\title{
On the numerical modelling of frozen walls in a molten salt fast reactor
}

\author{
Gregory Cartland Glover, Alex Skillen, Dzianis Litskevich, Stefano \\ Rolfo, David R. Emerson, Bruno Merk and Charles Moulinec
}

\section{Published version information}

Citation: G Cartland-Glover et al. 'On the numerical modelling of frozen walls in a molten salt fast reactor.' Nucl Eng Des, vol. 355 (2019): 110290. Is in proceedings of: 7th Workshop on the CFD for Nuclear Reactor Safety (CFD4NRS), Shanghai, China, 46 Sep 2018.

DOI: $10.1016 /$ i.nucengdes.2019.110290

C2019. This manuscript version is made available under the CC-BY-NC-ND 4.0 Licence.

This version is made available in accordance with publisher policies. Please cite only the published version using the reference above. This is the citation assigned by the publisher at the time of issuing the AAM. Please check the publisher's website for any updates. 


\title{
On the numerical modelling of frozen walls in a molten salt fast reactor th
}

\author{
Gregory Cartland Glover, ${ }^{\mathrm{a}, *}$, Alex Skillen ${ }^{\mathrm{a}}$, Dzianis Litskevich ${ }^{\mathrm{b}}$, Stefano \\ Rolfo $^{\mathrm{a}}$, David R. Emerson ${ }^{\mathrm{a}}$, Bruno Merk ${ }^{\mathrm{b}}$, Charles Moulinec ${ }^{\mathrm{a}}$ \\ ${ }^{a}$ Scientific Computing Department, Science and Technology Facilities Council, Daresbury \\ Laboratory, SciTech Daresbury, Keckwick Lane, Warrington, Cheshire WA4 4AD, United \\ Kingdom \\ ${ }^{b}$ Department of Mechanical, Materials and Aerospace Engineering, School of Engineering, \\ University of Liverpool, Liverpool L69 3BX, United Kingdom
}

\begin{abstract}
Coupled neutronic and thermal fluid dynamic simulations of a molten salt fast reactor have been performed. The reactor is an innovative concept intended to burn spent nuclear fuel. A key issue with the development of reactor concepts is the corrosive nature of the selected molten salts based on eutectics of lithium fluoride.
\end{abstract}

This paper investigates the usage of a frozen salt film to protect the vessel wall. Six case studies examine each of the modelling techniques that are applied for the simulation of frozen salt films on the walls of a molten salt fast reactor. The preliminary findings are that a very thin layer of frozen salt

\footnotetext{
${ }^{2}$ A data repository of the figures and tables provided in this article is available at the reference (Cartland-Glover et al. 2019).

${ }^{*}$ Corresponding author

Email address: greg.glover@stfc.ac.uk (Gregory Cartland Glover)

URL: https://www.scd.stfc.ac.uk/Pages/Computational-Engineering.aspx

(David R. Emerson)
} 
can form at a wall with isothermal conditions, but it is unstable in regions where high temperatures are found. Simulations of conjugate heat transfer in two-dimensions showed that the thin frozen salt films are unable to resist the heat flux resulting from the nuclear reactions.

Keywords: Molten Salt Fast Reactor, Neutronics, Monte Carlo, Nodal

Diffusion, Few Energy Groups, Computational Fluid Dynamics, Code-Coupling

\section{Introduction}

Molten salt fast reactors (MSFRs) are being developed to improve the sustainability, economics, safety and reliability along with proliferation resistance and physical protection of nuclear fission reactors . Recent studies have shown that MSFRs may be a sustainable option when spent nuclear fuel (SNF) is burnt (Fiorina et al. 2013; Heuer et al., 2014; Merk and Litskevich, 2015; Merk et al., 2017). The burning of SNF will significantly reduce the amount of waste passed to long term storage and the cost and complexity of the fuel cycle associated with fuel processing, as it will be limited to two steps: dissolution of the SNF fed to the reactor and extraction of the undesired fission products. It is envisaged that fuel processing will be operated continuously on site, which in turn can reduce site inventories and therefore improve site safety and protection.

A key problem with molten salt reactors (MSRs) since they were first developed in the mid-1940s by Oak Ridge National Laboratory (ORNL) in the aircraft propulsion experiment and followed by the molten salt reactor experiment (MSRE) programmes, (MacPherson, 1985), has been the corrosive 
nature of the fluoride salts that carry the nuclear fuel. Hastelloy $®$ was developed to resist corrosion of the MSRE reactor vessels exposed to high temperatures in the core region; however, to obtain significant reductions in the plutonium content of the fuel-salt eutectic used, operational lifetimes in excess of 60 years are required (Aufiero et al., 2013; Merk and Litskevich, 2015), which is far in excess of current MSR/MSFR concepts.

Fluoride salt corrosion of the vessel wall is compounded by a high neutron flux at the MSFR vessel wall (Merk and Konheiser, 2014). The neutrons will react with various isotopes in the nickel alloy, which may form helium and lead to the embrittlement of the vessel material as helium migrates through the grain boundaries. There has been significant research effort into modifying the composition of Hastelloy $(\mathbb{B}$ to improve its resistance to the effects of corrosion and neutron embrittlement (Ignatiev and Surenkov, 2013).

During the MSRE program, studies of the continuous fluorinators that separate uranium from the salt have demonstrated the potential of frozen salt films to protect vessel materials from the corrosive nature of the molten salts (Hannaford and McNeese, 1969). Therefore, a thin film of frozen salt at the core vessel wall of a molten salt fast reactor may protect the wall from the corrosive action of the molten fuel salt eutectic. The numerical studies described here investigate the feasibility of freezing of the fuel-salt at the core vessel wall. The fuel-salt eutectic studied here is lithium fluoride, plutonium fluoride, with the composition given in Table 5.2 of Gill (2016), and natural uranium which is used to demonstrate the possibility of using SNF Merk and Litskevich (2015). The numerical models used will have to account for the following effects: 
- The heat released by all neutrons upon the fluid dynamic models of MSFRs. The neutronics are strongly dependent on the temperature of the molten fuel-salt. Fluid expansion moves the isotopes further apart leading to a reduced probability that there will be a nuclear reaction and they are more likely to escape or leak from the reactor. The nuclear Doppler Effect occurs as the resonance peaks region widen due to increased oscillations of atomic nuclei. Consequently molten salt fast reactors have a strongly negative reactivity coefficient due to the influence of fluid expansion and the Doppler effect. As the fast neutrons either leak from the system or are captured and as only a very small proportion of neutrons are moderated by the molten salt, there is a lower density of neutrons at the thermal end of the neutron spectrum. The studies of Betzler et al. (2017), Brovchenko et al. (2019) and Zhang et al. (2019) provide an up-to-date overview of the state-of-the-art of the neutronic modelling of MSFRs.

- The influence of the neutronics on the fluid dynamics. Several approaches were taken to couple the neutronics to computational fluid dynamics depending on how the power density and thermophysical data are transferred between the codes used. The slowest approach is to write data to a file in a format the other code can read Yan et al. 2011), faster methods involve using message passing protocols controlled by a third program to communicate the data (Grahn et al., 2017; Pérez Mañes et al., 2014), another approach uses header files to provide the message passing and interpolation protocols, which can be compiled into both neutronics and fluid solvers at runtime (Tang et al., 2015). 
Mahadevan et al. (2014) coupled the neutronics and fluid solvers by exchanging data and converging both physics at each inner loop iteration. Alternative approaches implement a deterministic method into the fluid dynamics solver, but solve the neutronics and fluid dynamics on separate meshes (Fiorina et al., 2012; Laureau et al., 2013, 2015; Jareteg et al., 2015). Another approach also includes the fluid dynamics solver to access the neutronics solver as an external library Grahn et al., 2015). Note that Aufiero et al. (2014); Cervi et al. (2019) used the same meshes for to solve both the fluid dynamics and the neutronics in a Picard iteration. Other approaches to model the neutronics and thermal fluid hydraulics of MSRs have used specially prepared solvers such as Dalton-MSR (van der Linden, 2012) or COUPLE (Zhang et al. 2017) or thermal hydraulics codes and neutronics codes such as TRACE and PARCS (Pettersen, 2016), DYN3D and FLOCAL (Křepel et al. 2007), or DRAGON (Zhang et al., 2008). These approaches have been used irrespective of whether the neutronics have used stochastic (Monte Carlo techniques or transport equations) or deterministic (nodal diffusion equations or transport equations) methods.

- Model the melting and solidification of the fuel-salt eutectic. This will account for the change in physical properties and the sink in both the momentum equations and the equations for the transport of turbulence as well as the energy released or bound by melting or solidification. The approach used here applies a temperature dependent porosity Voller and Prakash, 1987). An alternative method that has explored the crystal growth of fluoride salt eutectics and the impact that the eutectic 
mixture has on the crystal structure formed with banding and spot formation of eutectic components (Tano et al., 2017). The scale of the crystal bands is of the order of $10 \mu \mathrm{m}$ and the resolution of the numerical models applied theirin were at a scale far below that in the engineering simulations performed here to test the feasibility of the frozen wall phenomena. Accounting for the distribution of the salt components would require sophisticated multi-scale and multi-physics simulations to provide fundamental phenomena. The specific physics simulations could then either be coupled to larger scale simulations or provide information on the salt composition (including fuel and fission products), the distribution of the local physical properties and the impact that they have on the neutronics as well as the transport of heat.

- Model the conjugate transfer of heat from the liquid through the frozen salt film to the solid wall. This will determine the feasibility of freezing the salt at the vessel wall by showing how is removed.

In the next section $(\S 2)$, descriptions of the numerical methods used to solve the neutronics, conjugate heat transfer with the melting and solidification of the salt as well as the coupling procedure are given. The subsequent section contains six case studies examining the application of the numerical models (§3). Finally we give our recommendations for future work $(\S 44$.

\section{Numerical Methods}

Here we describe the numerical models for calculations based on neutronics

$(\S 2.1)$ and the thermal fluid dynamics $(\S 2.2 \S 2.5)$ of a molten salt fast reactor, 
where conjugate heat transfer $(\S 2.4)$ and freezing of the salt $(\S 2.5)$ are take into account. The physical properties of the fuel-salt are described in $\S 2.6$ and the coupling between the neutronics and the fluid dynamics solvers are outlined in $\S 2.7$.

\subsection{Neutronics}

Two approaches were used to generate the models of the neutronics of the molten salt fast reactor core. The first approach is the stochastic Monte Carlo method, which is used to solve the Boltzmann equation for neutron transport (see Eq. 1).

$$
\frac{1}{v} \frac{\partial}{\partial t} \psi(\mathbf{r}, \hat{\mathbf{\Omega}}, E, t)+\hat{\mathbf{\Omega}} \cdot \nabla \psi(\mathbf{r}, \hat{\mathbf{\Omega}}, E, t)+\Sigma_{T O T}(\mathbf{r}, E) \psi(\mathbf{r}, \hat{\mathbf{\Omega}}, E, t)=S_{N}(\mathbf{r}, \hat{\mathbf{\Omega}}, E, t)
$$

Here $\psi$ is the angular neutron flux and it is a function of $\mathbf{r}$, the neutron position vector, $\boldsymbol{\Omega}$ direction vector, $E$, the neutron energy and, $t$, time. The remaining

terms are $S_{N}$, a general neutron source term, $\Sigma_{T O T}$, which represents the macroscopic total cross-section of the medium and, $v$, the neutron velocity, which is a function of $E$.

The Monte Carlo method utilises a pseudo-random number generator to simulate the characteristics (position, direction and energy) of each neutron, as it undergoes a number of collisions at different locations during its lifetime (i.e. from generation to leakage or capture) via an integral form of the neutron transport equation, Eq. (2), (Hérbert, 2009).

$$
\psi\left(\mathbf{r}, V_{n}, \hat{\Omega}, t\right)=\int_{0}^{\infty} d s \mathrm{e}^{-\tau\left(\mathbf{s}, V_{n}\right)} S_{D}\left(\mathbf{r}-\mathbf{s} \hat{\Omega}, V_{n}, \hat{\Omega}, t-s / V_{n}\right)
$$

Here $\mathbf{s}$ is distance a neutron has travelled from the source reference point $\mathbf{r}$ at a velocity of $V_{n}$, while $\mathrm{e}^{-\tau\left(\mathbf{s}, V_{n}\right)}$ is an integration factor and $S_{D}$ is the 
source density. The neutron cross sections applied in Monte Carlo solvers are obtained from fundamental and zero-power light water reactor experiments (Santamarina et al., 2014) and are interpreted as the probability that a collision of a given type will occur, this may include scattering, capture and fission amongst others. As a mainly meshless method, complex geometries can be used in Monte Carlo methods; however, accurate solutions of the neutronics require large numbers of particle histories resulting in long calculation times. The second approach uses the nodal diffusion method by applying the diffusion approximation that reduces Eq. (1) to a more simple differential equation, Eq. (3), which resolves the neutron flux isotropically with $G$ scalar equations for $G$ neutron energy groups (Duderstadt and Hamilton, 1976).

$$
-\nabla D_{g} \cdot \nabla \phi_{g}(\mathbf{r})+\Sigma_{t g} \phi_{g}=\sum_{g^{\prime}=1}^{G} \Sigma_{s, g^{\prime} g} \phi_{g^{\prime}}+\zeta_{g} \sum_{g^{\prime}=1}^{G} \nu_{g^{\prime}} \Sigma_{f, g^{\prime}} \phi_{g^{\prime}}+S_{g}
$$

Here $D_{g}$ is the $g$ th neutron diffusion coefficient and $\phi_{g}$ is the scalar neutron flux for the $g$ th neutron energy group, while $\Sigma_{t, g}, \Sigma_{f, g}$ and $\Sigma_{s, g^{\prime} g}$ are the total, fission and scattering neutron cross sections, where the neutrons scatter from group $g^{\prime}$ to group $g$. The fission source for the $g$ th group is $S_{g}$, while $\zeta_{g}$ is the fission spectrum of group $g, \nu_{g}$ is the number of neutrons per fission in the gth group.

Nodal diffusion codes differ in that the simplified system of equations can be solved by dividing the reactor core into a set of nodes. The material properties in terms of the homogenized cross section are assumed constant at each node, and the shape of the neutron flux is approximated within each node, for example, by polynomial functions.

To simulate the neutronics of the MSFR with the nodal diffusion code, DYN3D- 
MG (Kliem et al., 2016; Rohde et al., 2016), neutronics calculations of the full-core MSFR were first performed using version 2.1.29 of the Monte Carlo Transport code SERPENT (Leppänen et al., 2015) in order to determine the reactor criticality and to prepare the homogenized macroscopic cross-sections that are supplied to the MSFR model, which was then solved in the steady state solver of DYN3D-MG. The homogenized macroscopic cross-sections are discretized into 27 neutron energy groups based on the ANL distribution of energies (Leppänen, 2017). The macroscopic cross-sections are interpolated on a node-wide basis to assess the neutron interaction probability per unit length travelled and in general they depend on several physical parameters; however, only two parameters are considered here: the temperature and the density of the salt. The composition of the fuel salt eutectic $\left(\mathrm{LiF}-\mathrm{PuF}_{3}-\mathrm{UF}_{4}\right)$ of 77.5:4.4:18.1 (\% mol) was selected as the critical composition at a reactor inlet temperature of $623 \mathrm{~K}$ (Cartland-Glover et al., 2018b). Descriptions of the models implemented to solve the neutronics of the MSFR described here are also given in (Cartland-Glover et al., 2018b). The simulations performed by SERPENT also allow the benchmarking of the MSFR model applied in DYN3D-MG.

Note that when a steady or quasi-steady flow field is formed and that the distribution of the precursors is fully mixed, the impact of the delayed neutron precursors on the power distributions have been reported as two orders of magnitude less than the power distribution derived from the fission process (van der Linden, 2012; Pettersen, 2016). Therefore, the drift delayed neutron precursors were not modelled by either DYN3D-MG or SERPENT in the steady-state mode. Gamma heating was also not considered in the model as 
the neutron cross sections used by SERPENT were not of a sufficient quality to be considered for this feasibility study (Santamarina et al., 2014).

\subsection{Thermal fluid dynamics}

To model the thermal advection and convection of the Newtonian molten salt in an MSFR, the Reynolds-Averaged Navier-Stokes-Fourier equations given in Eq. (4) to Eq. (6), are applied.

$$
\begin{aligned}
\frac{\partial \rho}{\partial t}+\nabla \cdot(\rho \mathbf{u}) & =0 \\
\frac{\partial \rho \mathbf{u}}{\partial t}+\nabla \cdot(\rho \mathbf{u} \otimes \mathbf{u}) & =\nabla p+\nabla \cdot \tau+\rho \mathbf{g}+\nabla \cdot(\rho \mathbf{R})+\mathbf{S}_{\mathbf{u}} \\
\frac{\partial \rho c_{p} T}{\partial t}+\nabla \cdot\left(\rho c_{p} \mathbf{u} T\right) & =\nabla \cdot(\lambda \nabla T)-S_{T}+S_{T, \text { core }}
\end{aligned}
$$

where $\mathbf{u}$ is the Reynolds averaged fluid velocity, $\rho$, the density, $p$, the pressure, g, the gravity vector, $c_{p}$, the specific heat capacity at constant pressure, $\lambda$, the thermal conductivity and, $T$, the temperature. The phase-change related source terms for the momentum and energy equation are $\mathbf{s}_{\mathbf{u}}$ and $S_{T}$, respectively, and their implementation is detailed in $\S 2.5$. The energy equation also has an additional source term, $S_{T, c o r e}$, which supplies the heat released from the nuclear reactions.

For a Newtonian fluid of dynamic viscosity, $\mu$; $\tau$, is proportional to the rate-of-strain stress tensor with $\mathbf{I}$ being the identity matrix in Eq. (7), while the Reynolds stress, R, uses a linear eddy viscosity correlation in Eq. (8).

$$
\begin{aligned}
\tau & =\mu\left(\nabla \mathbf{u}+\nabla \mathbf{u}^{T}-\frac{2}{3}(\nabla \cdot \mathbf{u}) \mathbf{I}\right) \\
\rho \mathbf{R} & =-\mu_{T}\left(\nabla \mathbf{u}+\nabla \mathbf{u}^{T}-\frac{2}{3}(\nabla \cdot \mathbf{u}) \mathbf{I}\right)+\frac{2}{3} \rho k \mathbf{I}
\end{aligned}
$$


where $k$ is the turbulent kinetic energy and $\mu_{T}$ is the turbulent viscosity. The turbulence model for the closure of Eq. (5) via Eq. (8) will be discussed in $\S 2.3$.

Simulations were performed using version 5.0.5 of Code_Saturne, an open source CFD code developed by EDF R\&D France (Archambeau et al., 2004, Fournier et al., 2011). The software uses an unstructured finite volume colocated discretisation. The velocity/pressure coupling follows a SIMPLEC algorithm and Rhie and Chow interpolation is used to prevent the formation of checker-board oscillations. Second-order central differencing was used to discretise the convective and diffusive terms for the momentum equation, whereas second-order linear upwind has been used for the temperature. Firstorder up-winding was applied to the turbulence variables.

Simulations have been performed using an incompressible and dilatable unsteady algorithm in order to take into account variation of the physical properties, $\rho, \mu, c_{p}$ and $k$, while still retaining the advantages of an incompressible solver. The variation of physical properties as only functions of temperature are given in $\S 2.6$.

The simulations were performed in the transient mode with a gradient procedure based on the least squares method applied to a partially extended neighbourhood of the cell that eliminated checkerboarded solutions in poorly meshed regions. Variable time stepping procedures were used and the time step size was controlled by the Courant $(\leq 10)$ and the Fourier numbers $(\leq 100)$. 


\subsection{Turbulence}

A four equation, eddy viscosity RANS turbulence model, known as the Billard-Laurence- $v^{2} / k$ or BL- $v^{2} / k$ model (Billard and Laurence, 2012), was used to close the system of equations and compute $\mu_{T}$. In addition to solving the transport equations for the turbulent kinetic energy and the homogenous turbulent dissipation rate, the BL- $v^{2} / k$ turbulence model solves an extra transport equation for the dimensionless wall normal fluctuations, $v^{2} / k$, and an elliptic equation for the dimensionless wall distance, $\alpha$. Curvature correction has also been applied to the relevant turbulence model coefficient, $C_{\varepsilon 2}$, with the use of equation (28) of Cazalbou et al. (2005). By solving additional transport equations for $v^{2} / k$ and $\alpha$, and recasting both the transport equations for $k$ and $\varepsilon$, the near wall behaviour of the instantaneous wall normal fluctuations is improved along with reductions in the dependence of the model coefficients and the turbulent viscosity on the turbulent Reynolds number $R e_{T}$. Homogenuous and wall fluctuation functions are applied via the transport equation for $v^{2} / k$ that account for the redistribution of the dimensionless wall normal fluctuations by pressure fluctuations in the sublayers of the (Tunstall et al., 2014). This lead to improvements in the prediction of the defect layer for channel and separated flows while avoiding the use of wall damping functions (Billard and Laurence, 2012, Tunstall et al., 2014). The consequence of a better modelling of the boundary layer is that we can characterize the effect that sublayers in boundary layer have on the formation of a frozen salt film. This compares to either an LES or a low Reynolds number $k-\varepsilon$ turbulence model, which may have limited application at Reynolds numbers of $O\left(10^{6}\right)$ in the reactor core region. This will certainly allow us to resolve 
the boundary layer at a lower resolution than in the case of an LES model at such a high $R e$.

\subsection{Conjugate heat transfer}

The method used here to characterise the conjugate heat transfer required the use of fluid and solid domains within the fluid dynamics solver (Flageul, 2016). In the region where solid phases are present the convective term, $\nabla \cdot\left(\rho c_{p} \mathbf{u} T\right)$, in the energy equation, Eq. (6), is assumed zero. A thin wall boundary condition is used to define the interface between the fluid and solid regions, where mixed Neumann and Dirichlet conditions are applied to the fluid side of the thin wall and a Dirichlet condition to the solid side.

\subsection{Porous model for frozen salt films}

The source terms $S_{u}$ and $S_{T}$ for the solid-liquid phase change have been modelled according to the method presented in Voller and Prakash (1987). The method uses a porous media approach to model the solid and the mushy regions where appropriate source terms are devised to gradually suppress the velocity in the solid area and to account for the latent heat. From Darcy's law it is possible to derive the following formulation for the source term in the momentum equation, Eq. (5):

$$
\mathbf{S}_{\mathbf{u}}=-\frac{-C(1-\chi)^{2}}{\chi^{3}+q} \mathbf{u}
$$

where $\chi$ is the liquid fraction and varies from 0 (solid) to 1 (liquid) and $0<\chi<1$ is the mushy area. $C$ depends on the morphology of the porous media; however, we assume a value of $C=1 \times 10^{10}$ to ensure that the momentum is reduced to zero in regions where the solid is present. A value of $q=$ 
$1 \times 10^{-6}$ is applied to avoid any division by zero errors in solving the source term when $\chi$ is also zero. In the mushy area, the liquid mass fraction, $\chi$, is assumed to be a linear function of the temperature in terms of the liquidus, $T_{l}$, and solidus, $T_{s}$, temperatures, $\chi=\left(T-T_{s}\right) /\left(T_{l}-T_{s}\right)$.

The main uncertainties in the phase change model are introduced by the coefficients $C, q$ and the assumption of a linear dependency of the porosity with temperature. In setting up the model, we assume that the heat flux in the free flowing is sufficient for it to remain in the molten state and that the only regions where the salt can freeze is near to a wall in a region that should be cooler than the solidus point of the salt. We also assume that the fluid is Newtonian down to the solidus temperature and that any shear stress in the viscous boundary layer is linearly correlated. The determination of the value for each of these parameters and trends would require an experimental study for the materials applied here, as well as an assessment of both the solid phase and liquid phase properties over a large range of temperatures. Note that any neutron reaction occuring in the region of the thin frozen film for the coupled calculations was assumed to occur at the temperature of the nearest neighbouring node. The coarse resolution of the nodal diffusion code means that it is less likely to find any computational cell where the solid phase is present.

For consistency with the momentum transport equations, Eq. (5), source terms of a similar form to that in Eq. (9) are also applied to the turbulence transport equations for $k, \varepsilon$ and $v^{2} / k$, where $\mathbf{u}$ is replaced by the corresponding turbulence parameter. The sign and coefficients applied to the source term remain unchanged. The Helmholtz equation for the parameter 
$\alpha$ is an exception, as it expected that this term should not influence the non-dimensional distance from the domain wall. Therefore, the turbulent source term is not applied the $\alpha$ parameter.

The latent heat $(L)$ dependent source term for the energy equation, Eq. (6) is composed by a time dependent term that reads:

$$
S_{T}=\frac{\partial(\rho \chi L)}{\partial t}
$$

In the time term, the liquid mass fraction is re-cast as a function of $T$, which allows the trapezoidal rule to be used to solve implicitly the relevant terms. The convective term is, on the other hand, solved with a fully first-order UPWIND scheme.

\subsection{Physical properties of the phases}

The physical properties of the fluid form of the fuel-salt eutectic are based on the lithium thorium fluoride eutectic $\left(\mathrm{Li}_{3} \mathrm{ThF}_{7}\right)$ reported by Rouch et al. 2014). The functions of the physical properties for the fluid phase defined are the density, viscosity, thermal conductivity and the specific heat capacity, which are given between Eq. (11) and Eq. (14). All of these equations were applied to the dilatable Reynolds averaged Navier-StokesFourier equations given in $\$ 2.2$ to account for the variation in viscosity and specific heat capacity, which have a much larger change in value compared to the density over the range of temperatures considered. Note that the fluid properties were extrapolated outside of the range of validity and that the liquid 
and mushy phases behave in a Newtonian manner.

$$
\begin{array}{rlrl}
\rho_{f}=4983.56-0.882 T & 893 \mathrm{~K}<T<1,123 \mathrm{~K} \\
\mu=5.55 \times 10^{-8} \rho e^{3689 / T} & 898 \mathrm{~K}<T<1,119 \mathrm{~K} \\
c_{p, f}=-1111+2.78 T & 867 \mathrm{~K}<T<907 \mathrm{~K} \\
k_{f}=0.928+8.4 \times 10^{-5} T & 891 \mathrm{~K}<T<1,020 \mathrm{~K} \\
c_{p, s}=\frac{1 \times 10^{3}}{M_{w, L i 3 T h F_{7}}}\left(252.1+5.730 \times 10^{-2} T\right. & T<814.22 \mathrm{~K} \\
& \left.+1.514 \times 10^{-6} T^{2}-2.962 \times 10^{-6} T^{-} 2\right) &
\end{array}
$$

The solid phase properties are also based on $\mathrm{Li}_{3} \mathrm{ThF}_{7}$ for the specific heat capacity given in Capelli (2016), where $M_{w, L i_{3} T h F_{7}}$ is the molecular weight of the salt in Eq. (15). The solid phase density of $\mathrm{Li}_{3} \mathrm{ThF}_{7}$ was taken as $4,330.09 \mathrm{~kg} \mathrm{~m}^{-3}$ (Catalyst Database, 2017); however, the thermal conductivity $\left(5.61 \mathrm{~W} \mathrm{~m}^{-1} \mathrm{~K}\right)$ is based on $\mathrm{LiF}$ at $800 \mathrm{~K}$ due to a lack of appropriate literature data (Janz et al., 1979).

The latent heat of fusion, $L$, of $\mathrm{Li}_{3} \mathrm{ThF}_{7}$ is defined as $151.58 \mathrm{~kJ} \mathrm{~kg}^{-1}$ with an error of $\pm 0.54 \mathrm{~kJ} \mathrm{~kg}^{-1}$, which is used to determine the heat conversion per kilogram of material melted or solidified. This value for the latent heat of fusion was obtained from the value reported by Gilbert (1962).

Gilbert (1962) also reported the variation in the fraction of salt melted against temperature. The solidus and liquidus temperatures of $\mathrm{Li}_{3} \mathrm{ThF}_{7}$ were estimated as $814.22 \mathrm{~K}$ and $841.55 \mathrm{~K}$. They were obtained using the equation of the curve $\chi=(1 /(m T+c))^{1.5}$ with the coefficients $m=0.744568 \pm 0.01817 \mathrm{~K}^{-1}$ and $c=627.786 \pm 15.25$. The function may also be used in the application of the porous model for the melting and solidification, though the linear function was used in the simulations performed here. 


\subsection{Coupling Interface}

The coupling procedure implemented here uses a $\mathrm{C}++$ library called the Multiscale Universal Interface, MUI, (Tang et al., 2015). The library is a lightweight set of interface commands and interpolation routines encoded as header files with only the Message Passing Interface (MPI) as an external dependency. This means that the calls to the library can be inserted into the relevant sections of the source code of each solver to fetch and push data with minimal alterations. The library communicates data on a nodal basis and is applicable to both Eulerian and Lagrangian reference frames. A number of adaptable interpolation routines are available to provide flexibility with regards to the reference frame and type of data fetched and pushed.

The interpolation routine used in the coupled simulations performed here is a scheme that finds the nearest corresponding node. Data is transferred in a staggered explicit strategy (Mahadevan et al., 2014, see Fig. 1a of), with temperature and power density exchanged at the beginning of each cycle and then the corresponding system of equations is solved. Code_Saturne is operated in the transient mode due to the unsteady nature of the flow concerned (MSFR core Reynolds number of the order of $10^{6}$ ).

DYN3D-MG was operated in the steady neutronics "feedback mode" without the internal thermal hydraulics module known as FLOCAL Kliem et al., 2016; Rohde et al., 2016). Therefore, we consider that DYN3D-MG is a loosely coupled neutronics "inner iteration loop", which is external to the time step iteration loop of Code_Saturne. 


\section{Case Studies}

Six case studies are described here, which present the verification of the numerical models $(\oint 3.1, \S 3.2, \S 3.3$ and $\S 3.5$ required to simulate a frozen salt film in a molten salt fast reactor $(\S 3.3$, $\S 3.4$ and $\S 3.6$.

\subsection{Verification of the neutronics models - cylindrical test case}

The simulations performed in this case study are an extension initial simulations performed with DYN3D-MG for the simulation of a molten salt fast reactor reported in Cartland-Glover et al. (2018b). Here we examine the impact that the change in temperature of the salt medium has on the neutronics solutions obtained by both Serpent and DYN3D-MG. The geometries plotted in Fig. 1 were selected, they were a cylindrical geometry (the mesh) and a configuration we refer to as MSFR-EVOL (the continuous grayscale fields), which corresponded to Geometry II reported by Rouch et al. (2014). MSFR-EVOL has a core region made from side walls in the shape of a hyperboloid and two inward facing cones forming the base and top walls with 16 primary circuits (not considered in the neutronics models) for heat exchangers, pumps and gas separation. The radius at the narrowest point on the hyperboloid was $1.06 \mathrm{~m}$ with a height of $1.6 \mathrm{~m}$ between the cone apices. The radius and the height of the cylinder corresponded to these dimensions. Note that the JEFF-3.1.1 nuclear data library (Santamarina et al., 2014) and the ANL 27 energy group structure (Leppänen, 2017) were used.

The MSFR-EVOL problem was solved using SERPENT in 17 calculations at different temperatures with densities calculated by Eq. (11) to prepare the cross sections for their use in the DYN3D-MG calculations. Two extra 

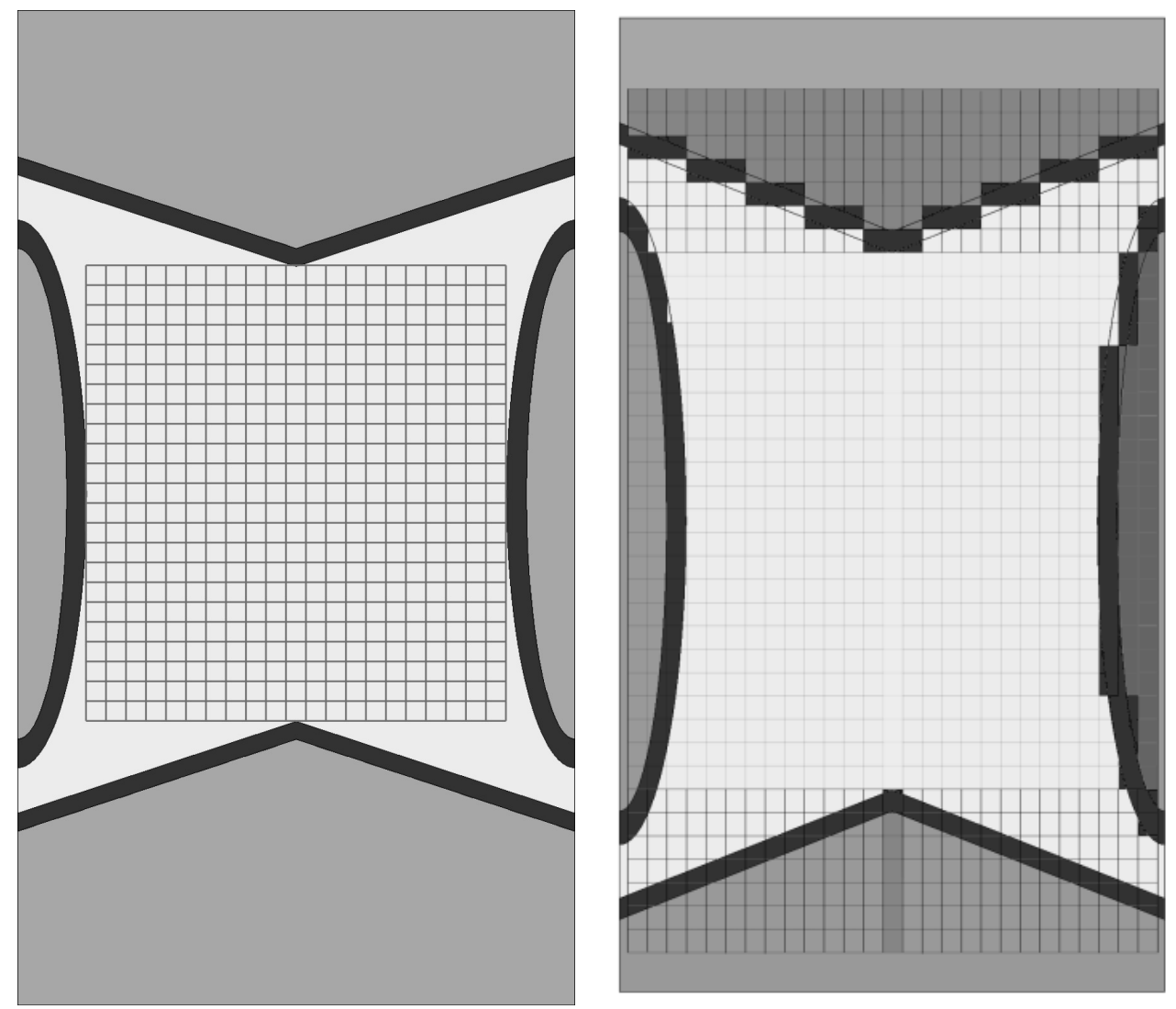

Fig. 1. Side view of the geometries used in SERPENT and DYN3D-MG meshes for the cylindrical geometry (left) and the approximation for the EVOL-optimised geometry (right). 
calculations at $973 \mathrm{~K}$ and $1,023 \mathrm{~K}$ were used to test the cross-section interpolation routines in DYN3D-MG. Note that for the SERPENT calculations with $8 \times 10^{5}$ particles per cycle for 2,000 cycles, the effective neutron multiplication factor, $k_{e f f}$, was accurate to $11 \mathrm{pcm}$ (percent mille) with a $3 \sigma$ confidence interval, where $\sigma$ is the standard deviation (see Fig. 2 for the trend in $\left.k_{e f f}\right)$. These neutron cross-sections were applied to the step-wise quasicylindrical geometry simulated with DYN3D-MG for nineteen temperatures. Two additional temperatures were considered were used to check that the interpolation procedures in DYN3D-MG produce physically relevant results. The core modelled in DYN3D-MG was constructed from 349 "homogeneous fuel assemblies" that were $10.09 \mathrm{~cm}$ wide by $6.956 \mathrm{~cm}$ high. Each fuel assembly was modelled by 23 "nodes". A DYN3D-MG node is a volume element across which the neutron flux and the interface currents are estimated. The step-wise quasi-cylindrical geometry is a crude approximation of the core and it resulted in a lower $k_{e f f}$ due to the cylinder having a smaller volume than the MSFR-EVOL and therefore a smaller fuel loading.

A second set of SERPENT calculations were performed for the cylindrical geometry with only 20,000 particles over 2,000 active cycles that gave $k_{\text {eff }}$ values that were accurate to approximately $66 \mathrm{pcm}$ with a $3 \sigma$ confidence interval (see error bars in Fig. 2) to test the accuracy of the DYN3D-MG cylindrical calculations in terms of the cross-sections applied. All of the DYN3D-MG cylinder calculations with cross-sections prepared using the MSFR-EVOL geometry were less than $0.25 \%$ different from the SERPENT cylinder calculations. Thus, it is considered that both the cross-sections and methodology used in DYN3D-MG are able to reproduce the change in the 


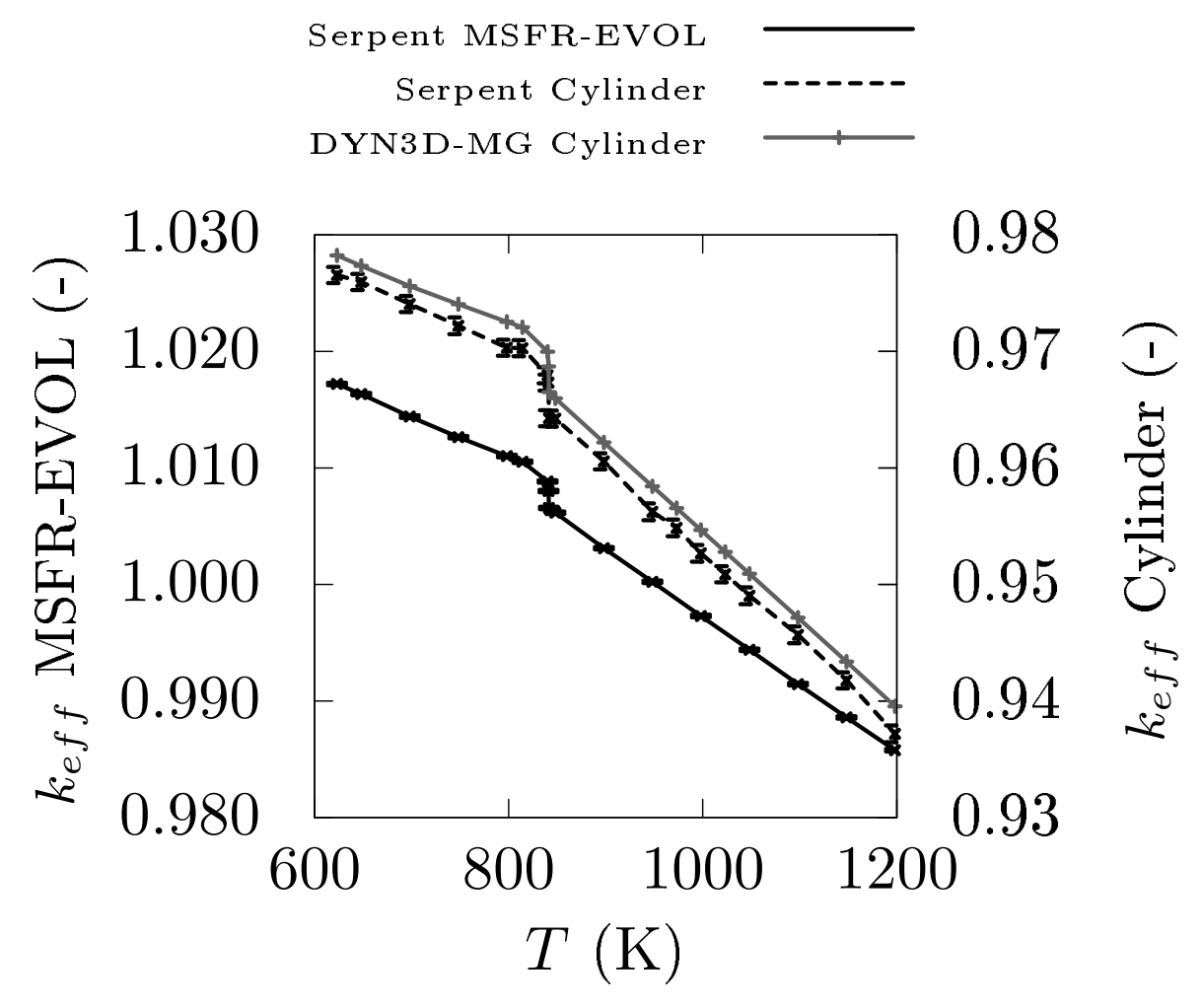

Fig. 2. Variation of the effective neutron multiplication factor, $k_{e f f}$, against temperature of the fuel-salt obtain from both of the neutronics solvers.

reactor state as the temperature is increased from a solid salt to a molten salt at a high temperature. The interpolation routines for the cross sections appear to be working appropriately, as the reactor states obtained at these temperatures is consistent with the trend of the reactor state plotted for both Serpent and DYN3D-MG in Fig. 2. 


\subsection{Benchmarking of temperature dependent porosity}

To verify the methodology used to simulate the solid-liquid phase change, the case of a differentially heated square cavity has been used as proposed by Voller and Prakash (1987). The test problem applies two vertical isothermal walls $\left(T_{H o t}=-T_{\text {Cold }}=0.5\right.$ and $\left.\Delta T=T_{H o t}-T_{\text {Cold }}=1\right)$ and two horizontal adiabatic walls, $\partial T / \partial y=0$. The edge on the cavity has a length equal to 1 with $x$ being the horizontal direction and $y$, the vertical. There is a uniform and equal distribution of cells in the $x$ and $y$ directions.

The flow is considered 2-D laminar, as the Rayleigh number is small $(10,000)$. The Prandtl and Stefan numbers are $\operatorname{Pr}=c_{p} \mu / \lambda=1,000$ and Ste $=$ $c_{p} \Delta T / L=5$, respectively. The solidus and liquidus temperatures are antisymmetric with respect to $T=0$ and take the values $T_{L}=-T_{S}=0.1$.

An analysis of the quality of the solution to the mesh density is carried out (see Table 1 and Table 2). Six difference mesh sizes were tested with

the first three using the same mesh resolutions as Voller and Prakash (1987). The solutions were progressed with time with a constant step size of 0.1 for $4 \times 10^{4}$ iterations.

Eight parameters were obtained from the solution at the last time step where a steady state solution had been achieved. The four of the parameters are given in Table 1 and they are the maximum of the velocity magnitude, max $\mathbf{u}$, the maximum Courant number, $\max C F L$, the non-dimensional first cell centre distance for momentum and temperature, $y^{+}$and $T^{+}$. The percentage difference of the maximum velocity magnitude did not become less than $1 \%$ until the number of cells was greater than or equal to 160 in the $x$ and $y$ directions. A consequence of this higher resolution is that $y^{+}<<1$ and 
Table 1. Mesh convergence of the square cavity detailing the maximum velocity, Courant number (CFL), $y^{+}$and $T^{+}$. The time step size was 0.1 time units for all resolutions.

\begin{tabular}{rrrrrrr}
\hline \multicolumn{2}{c}{ No. of cells } & max u & \% Diff. & $\max$ CFL & \multicolumn{1}{l}{$y^{+}$} & $T^{+}$ \\
$N_{x}$ & $N_{y}$ & & & & & \\
\hline 10 & 10 & 0.02513 & - & 0.012 & 0.0272 & 27.16 \\
20 & 20 & 0.01477 & -41.24 & 0.026 & 0.0113 & 11.27 \\
40 & 40 & 0.01058 & -28.39 & 0.057 & 0.0056 & 5.62 \\
80 & 80 & 0.01088 & 2.91 & 0.114 & 0.0028 & 2.84 \\
160 & 160 & 0.01093 & 0.39 & 0.228 & 0.0014 & 1.42 \\
320 & 320 & 0.01093 & -0.01 & 0.456 & 0.0007 & 0.71 \\
\hline
\end{tabular}

Table 2. Mesh convergence of the square cavity detailing the phase transistion points at $y=0$ and $y=1$ and $T= \pm \epsilon$, where $\epsilon=0.1$.

\begin{tabular}{cccccccccc}
\hline \multicolumn{3}{c}{ No. of cells } & \multicolumn{8}{c}{ Phase transition points } \\
$N_{x}$ & $N_{y}$ & $x_{T=-\epsilon \mid y=0}$ & $\%$ Diff. & $x_{T=\epsilon \mid y=0}$ & $\%$ Diff. & $x_{T=-\epsilon \mid y=1}$ & $\%$ Diff. & $x_{T=\epsilon \mid y=1}$ & $\%$ Diff. \\
\hline 10 & 10 & 0.577 & - & 0.853 & - & 0.118 & - & 0.219 & - \\
20 & 20 & 0.443 & -23.3 & 0.776 & -9.0 & 0.143 & 21.6 & 0.246 & 12.0 \\
40 & 40 & 0.380 & -14.2 & 0.713 & -8.2 & 0.179 & 24.6 & 0.285 & 15.8 \\
80 & 80 & 0.360 & -5.2 & 0.685 & -3.9 & 0.196 & 9.9 & 0.305 & 7.1 \\
160 & 160 & 0.355 & -1.5 & 0.674 & -1.5 & 0.202 & 3.0 & 0.312 & 2.3 \\
320 & 320 & 0.354 & -0.4 & 0.670 & -0.6 & 0.204 & 0.9 & 0.314 & 0.7 \\
\hline
\end{tabular}


$T^{+} \rightarrow 1$. The transition points between the liquid, mushy and solid phases on the upper and lower boundaries of the cavity are given in Table 2. The percentage change in the transition points between the phases at a resolution of 160 cells in the $x$ and $y$ directions were less than $2 \%$ at $y=0$ and $4 \%$ at $y=1$.

At a resolution of 160 cells the percentage change in the maximum velocity and the phase transition points dropped to below $1 \%$. This indicates that the mesh resolution must approach a $T^{+}$of 1 before mesh independent solutions are achieved. Fig. 3 compares the current results and the reference work of Voller and Prakash (1987). In the simulations performed with Code_Saturne the resolution of the grid used for the results presented in Fig. 3 was 320 by 320 cells. The general trends in both the temperature and liquid fraction are very similar, but there are differences on the bottom wall. These differences are mainly related to the position of the transition points between the different phases (solid-mushy-liquid). The transition points at $y=0$ are located at $x_{T=-0.1}=0.45$ and $x_{T=0.1}=0.73$ for the present work and at $x_{T=-0.1}=0.31$ and $x_{T=0.1}=0.85$ for Voller and Prakash (1987).

It is the authors opinion that the main reason of this difference, since the porous model uses the same constants $\left(C=1.6 \times 10^{3}\right.$ and $\left.q=1 \times 10^{-3}\right)$, has to be attributed to the different discretisation of the time term for the latent heat in the energy equation Eq. (6). In the present study, the temperature terms at the next time step were solved implicitly (as described in $\S 2.5$, whereas in Voller and Prakash (1987), an iterative method was used in order to have $\chi$ and $T$ synchronised, which resulted in the temperature derivative in the wall normal direction not being exactly zero. The advantage of the 


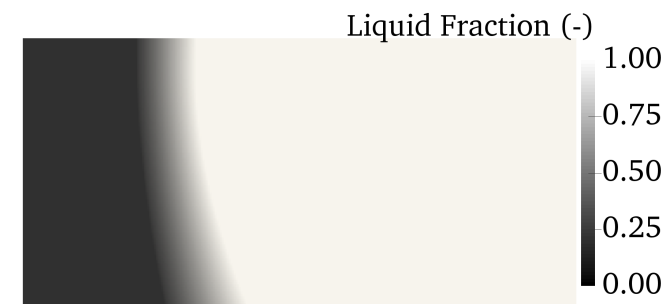

Voller and Prakash (1987): $40 \times 40$ cells

Current model: $320 \times 320$ cells

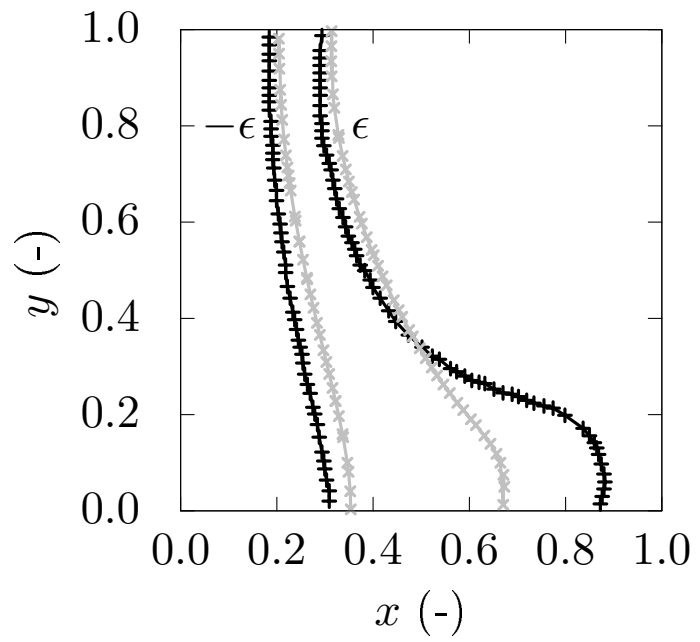

Fig. 3. Comparison between between results obtained by Code_Saturne with the study of (Voller and Prakash, 1987). The field at the top is the liquid fraction. The plot below gives the contours at $T= \pm \epsilon= \pm 0.1$, which indicate the boundaries of the mushy area. Note that one in every twenty data points is plotted for the $320 \times 320$ mesh. 
current discretisation can be seen in the temperature iso-lines on the bottom wall, where the temperature derivative in the normal direction is exactly 0 , as prescribed by the boundary condition. Moreover, the method described here is also suitable for sharp interface problems provided that a sufficiently fine mesh resolution is used.

However, in Voller and Prakash (1987), it is not clearly the case since the derivative for the lines at $T=0.1,0.05$ and 0.0 clearly shows a large angle with the normal direction. This should not only be a post-processing issue since the other iso-lines, in the liquid and solid zones, clearly have a homogeneous Neumann boundary condition. Alternative contributing factors in the difference between the solutions may be attributed to the resolution of the mesh ( 40 by 40 cells) and the maximum Courant number (10) of the simulations performed by Voller and Prakash (1987). The consequence of these effects is that the solution obtained by Voller and Prakash (1987) may not have a sufficient resolution to accurately capture the phenomena and that the transport of the solid film front moves across more than one cell per timestep. Note that a recent study (Rasendra, 2012) has tested solidification and melting models of Code_Saturne against a dedicated solidification solver (Ahmad et al. 1998) was able to replicate the evolution of the solid front over $500 \mathrm{~s}$ of simulation time. The evolution of the solid front was significantly less than that the front evolution in the case of Voller and Prakash (1987) and the evolution of the front in the simulations performed here (Fig. 3). Further work is required to validate these models for the case of turbulent convection in a cavity and for the evolution of a solid film in a channel flow. 


\subsection{Two-dimensional fluid dynamic calculations of an MSFR with frozen} salt films

\subsubsection{Problem description}

The thermal fluid dynamics simulation of the flow in a 2-D slice of the MSFR-EVOL configuration was performed where frozen salt films were taken into account. A 2-D mesh with a depth of one cell thick was used with symmetry conditions applied to the front and back surfaces extending from the core centre to the inlets and outlets (Fig. (4). The remaining surfaces were isothermal wall conditions at $623 \mathrm{~K}$, a zero gradient outlet condition, a symmetry condition at the core centre and an inlet condition.

The inlet condition applied profiles of the velocity components, the temperature and the turbulence parameters, which were obtained from prior simulations carried out assuming periodicity for an extrusion of the inlet and performed using Code_Saturne. The fully developed flow profile resulted in a mean inlet velocity of $3.82 \mathrm{~m} \mathrm{~s}^{-1}$ and a mean temperature of $898 \mathrm{~K}$. Note that the spatial function of the neutronic heat source, Eq. (16) was used to simulate the heating of the fluid in the core region (Rouch et al., 2014).

$$
\begin{aligned}
& s_{T, \text { core }}=\left\{\begin{array}{l}
Q \cos \left(\frac{\pi}{2} \cdot \frac{r_{n}}{r_{c, 0}}\right) \cos \left(\frac{\pi}{2} \cdot \frac{z_{c}}{z_{c, 0}}\right) \quad \leq 1.405 \mathrm{~m} \\
\left|0.1 * Q \cos \left(\frac{\pi}{2} \cdot \frac{r_{n}}{r_{c, 0}}\right) \cos \left(\frac{\pi}{2} \cdot \frac{z_{c}}{z_{c, 0}}\right)\right|>1.405 \mathrm{~m}
\end{array}\right. \\
& r_{n}= \begin{cases}r_{c}\left(1.05550+0.228960 z_{c}\right) & z_{c} \leq 0.59 \mathrm{~m}, \\
r_{c}\left(1.40277+0.359622 z_{c}\right) & z_{c}>0.59 \mathrm{~m},\end{cases}
\end{aligned}
$$

Here $r_{c}$ and $z_{c}$ are the radial and vertical positions in the core region. $Q$ is the maximum heating rate with a value of $7.8316907 \times 10^{8} \mathrm{~W} \mathrm{~m}^{-3}$, while $r_{c, 0}=$ $1.671773 \mathrm{~m}$ and $z_{c, 0}=1.8744 \mathrm{~m}$ are factors that non-dimensionalise the radial 


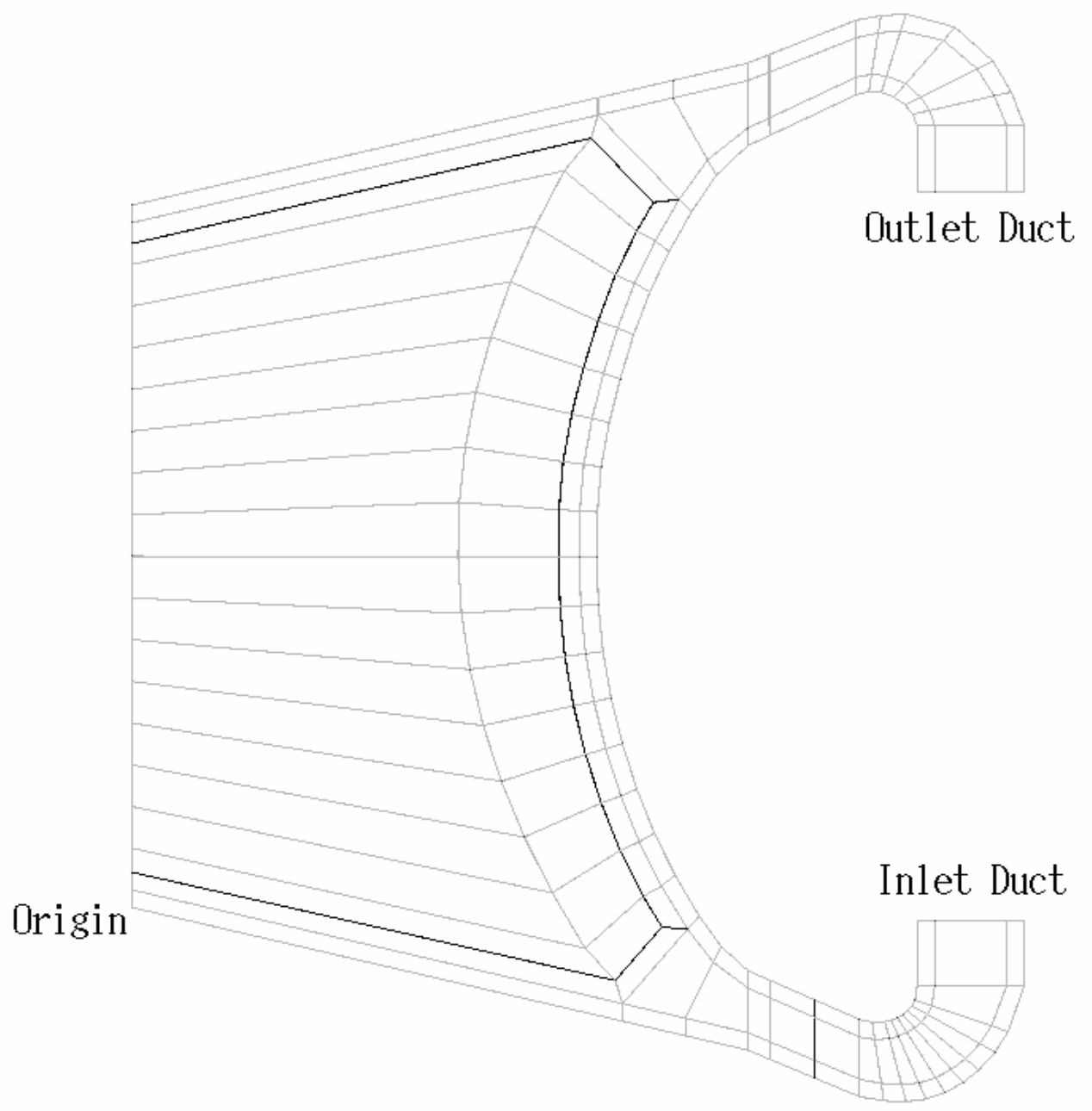

Fig. 4. Plots of the 2D plane domain and mesh used to represent the molten salt fast reactor. 
and vertical position. A reduction factor was applied to the core inlet and core outlet to mimic the effect of the 3D coupled simulations, which are discussed in $\S 3.6$, as the inlet and outlet ducts are not modelled by DYN3D-MG (see $\S 3.1$.

\subsubsection{Assessment of the near-wall mesh density}

Following the study in $\S 3.2$, a meshing strategy was selected that aimed to achieve a near-wall $T^{+}$as close to 1 as possible. This is a consequence of the inclusion of the solification models and the Prandtl number of the molten salt being between 10 and 23 leading to strong temperature gradients in the viscous sublayer. This means that the first cell-centre should be in the region where the temperature profile is in the linear molecular diffusion (viscous and thermal) sublayer of the boundary layer with the form, $T^{+}=P r y^{+}$in limit $y^{+} \leq 4 \mathrm{Pr}^{-1 / 3}$. The linear thermal sublayer region is limited to $y^{+} \leq 1.36$ and $y^{+} \leq 1.89$ over the range of temperatures observed in the simulations (Kader, 1981, 1991).

Therefore, to get a $T^{+}$close to 1 , it is required that the distance of first cell centre from the wall to be very small. This is unsuitable for a uniform size distribution of cells in a reactor geometry with a volume of $9 \mathrm{~m}^{3}$.

To effectively model the flow, we must increase the mesh density next to the wall region. This was achieved through the use of two layers of o-grids, a hexahedral block decomposition method, which was used to divide the mesh into regions (see Fig. (4). This block decomposition method enables a hexahedral mesh to be generated in the complex geometry found in the core region. One of the o-grids formed the near-wall layer, which was $40 \mathrm{~mm}$ thick and passed through the inlet, outlet and core symmetry conditions. The 
Table 3. Mean $y^{+}$and $T^{+}$for each 2D mesh of the molten salt fast reactor based on the EVOL-optimised geometry (Rouch et al., 2014).

\begin{tabular}{cccr}
\hline No of cells & First Node Wall Distance $(\mu \mathrm{m})$ & $\overline{y^{+}}$ & $\overline{T^{+}}$ \\
\hline 50300 & 100 & 8.23 & 133.52 \\
50300 & 50 & 3.70 & 69.74 \\
61986 & 25 & 0.64 & 10.82 \\
101872 & 10 & 0.29 & 1.25 \\
\hline
\end{tabular}

other o-grid is found in the inner blocks of the core region (see black lines in Fig. (4). The blocks that form the inlet and the outlet do not have a second o-grid level. The number of blocks used along the ducts and the core height was increased with the resolution of the mesh at the wall in order to obtain the best possible mesh quality.

Several meshes were used to determine the size of the first cell-centre from the wall to give a $y^{+}<<2.3$, which is the first cell centre limit for the BL- $v^{2} / k$ turbulence model and a small $T^{+}$; the mesh presented in Cartland-Glover et al. (2018a) and used here without a heat exchanger gave a $\max T^{+}$of about 30 (see Table 3).

A further mesh was created with a distance of $0.01 \mathrm{~mm}$ for the first cell with a hyperbolic expansion of the cells up to a size of $3 \mathrm{~mm}$ at a distance of $40 \mathrm{~mm}$ from the wall compared to the inlet width of $27 \mathrm{~cm}$ and narrowest core diameter of $2.12 \mathrm{~m}$. This gave a $102 \mathrm{k}$ cell hexahedral mesh with $y^{+} \leq 1$ and a fluctuating $T^{+}$that remained below $T^{+} \leq 10$ (see Fig. 5). Note that the for the first ten seconds of simulated time the freezing model was not applied 


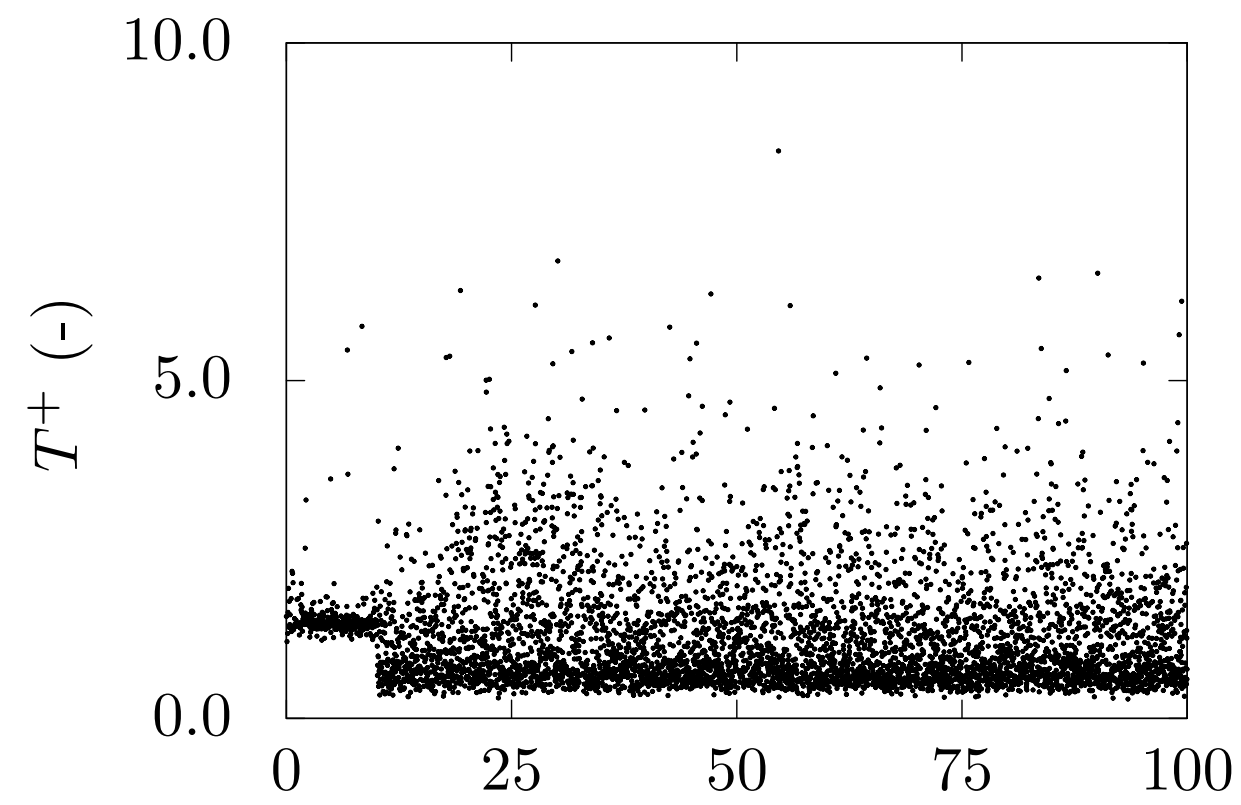

Fig. 5. Trace of the maximum $T^{+}$. The fluctuations in the maximum $T^{+}$ were recorded every 100 time steps. The depth of the first cell centre was $10 \mu \mathrm{m}$. 
and this is apparent with the higher $T^{+}$values during this time.

The maximum cell sizes of the $102 \mathrm{k}$ mesh were $20 \mathrm{~mm}$, which were located in the bends of the inlet and outlet ducts. Two regions of the mesh had a minimum cell angle of 38 degrees; they were located in the core region near to the inlet and outlet ducts where an o-grid boundary was located. The maximum volume change was less than 3 times that of the neighbouring cell; however, large aspect ratio elements were found near to the wall.

The time averaged radial profiles of temperature and velocity magnitude at the narrowest part of the reactor core barrel, where the radius, $R$, was $1.06 \mathrm{~m}$, are plotted in Fig. 6. The salt film was left to develop for 40 s once the freezing model was applied and the calculations were performed for a further $100 \mathrm{~s}$ for the lower resolution cases and $50 \mathrm{~s}$ for the $102 \mathrm{k}$ mesh where averages of the velocity and temperature were analysed.

The profiles of temperature show a similar tendency across the radius of the reactor core with lower temperatures in the centre and higher temperatures next to the isothermal wall. The cold jet from the core inlet can also be observed with the reduction in temperature along core centre.

Temperatures below solidus point were only observed with a first node wall distance below $25 \mu \mathrm{m}$. The only region where the temperature was observed below the solidus temperature was less than $80 \mu \mathrm{m}$ from the wall. This is a consequence of the instability of the frozen layer that keeps forming and vanishing, which highlights the necessary requirement to have an extremely fine near-wall mesh to be able to capture the frozen film under the very large heat flux coming from the core region.

A sharp rise in the temperature associated with the thermal boundary layer 

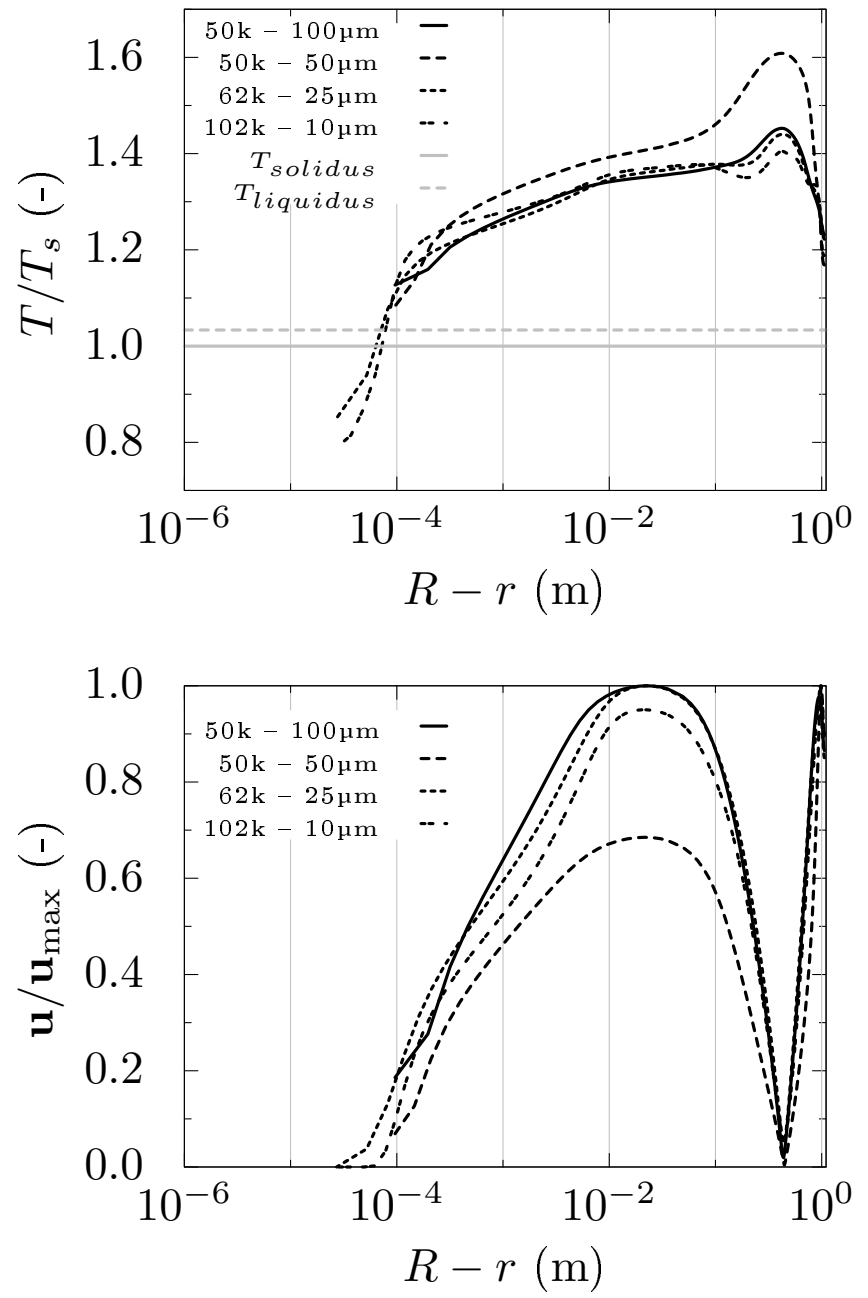

Fig. 6. Mid reactor radial profiles $(R=1.06 \mathrm{~m})$ of the mean temperature $(\mathrm{K})$ at the top and mean velocity $(\mathrm{m} / \mathrm{s})$ below. The profiles are respectively non-dimensionlised by the solidus temperature, $814.22 \mathrm{~K}$, and the maximum velocity. 
is observed between 0.2 and $0.5 \mathrm{~mm}$. The viscous boundary layer is observed at wall distances of between $0.1 \mathrm{~mm}$ and $100 \mathrm{~mm}$. Several mesh resolutions were tested and only the cases with a first cell centre distance below $25 \mu \mathrm{m}$ could capture the frozen salt film over more than a couple of cells in the core region.

\subsubsection{Model uncertainty}

A number of uncertainties are introduced into the model by the assumptions detailed in $\$ 2.2$ 2.3 and $\$ 2.5 \$ 2.6$, which included the selection of the physical properties of the eutectic salt modelled, the application of RANS turbulence models in transient mode with accuracy limited by the two scales considered that smooth the eddies formed and the distribution of the heat source applied to the fluid as well as the boundary conditions we apply to the inlet conditions and the walls. The validation of the approach used here requires comparison against experiments, which do not exist for the salt eutectic we have considered. However, we provide a crude initial approximation of the uncertainty of the 2-D MSFR model is based around the mean velocity and temperature profiles and the physical properties of the fluids. The Nusselt number could be determined for the range of uncertainty in the physical properties, following the methods of Sohal et al. (2010). We assume that the Dittus-Boelter correlation is valid $\left(N u=0.023 \operatorname{Re}^{0.8} \operatorname{Pr}^{0.4}\right)$, where the Reynolds number is defined as $R e=2 R u \rho / \mu$. We take the fluid velocity magnitude, $u$ and temperature to be the mean of the mid-plane time-averaged profile for the 102k mesh plotted in Fig. 6 and adjust the physical properties to the reported minimum and maximum errors (Ignatiev et al., 2012). The viscosity correlation is assumed to have an error of $\pm 3 \%$ following the study 


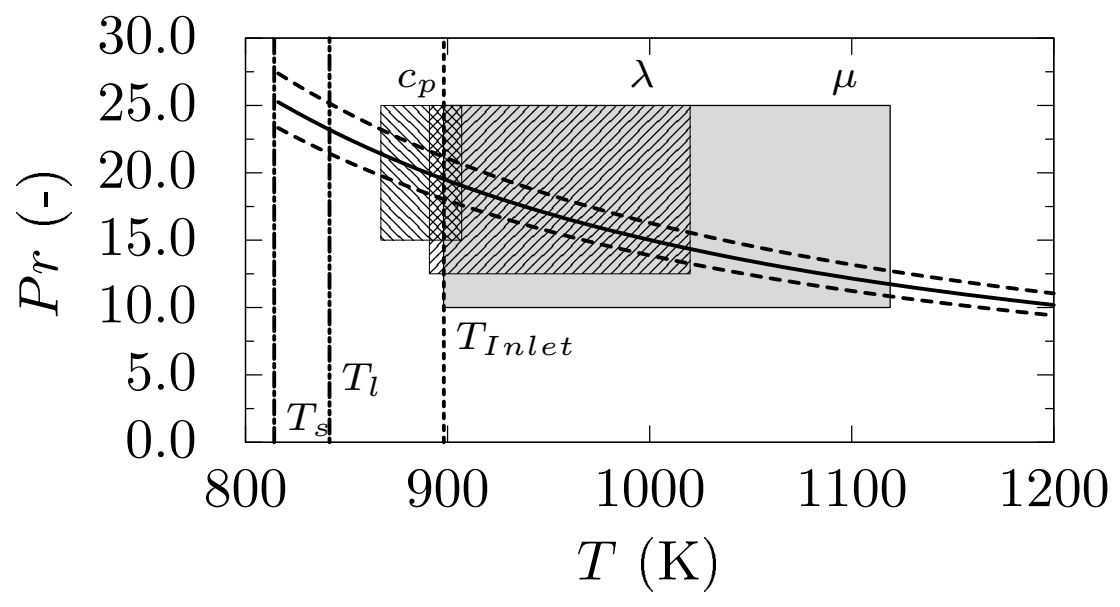

Fig. 7. Variation of the Prandtl number of the fuel-salt, $\operatorname{Pr}=c_{p} \lambda / \mu$, with temperature. The shaded boxes indicate the limit of interpolation and the dashed lines indicate the range of the maximum experimental errors.

of Heller et al. (2017).

To emphasize the uncertainty in the physical properties, the profiles in Fig. 7 indicate the maximum and minimum error of the Prandtl number estimated from the correlations of the fuel-salt eutectic between Eq. 12) and Eq. (15), as the temperature is increased from below the solidus temperature. The boxes in Fig. 7 indicate the limits regions of interpolations for the correlations for thermal conductivity, specific heat capacity and viscosity. It is therefore clear that a small region around the inlet temperature, $898 \mathrm{~K}$ is the only region where all correlations are interpolated. We assumed that the Prandtl number 
increases as the exponential function given in Fig. 7 as the temperature is reduced to the solidus temperature following the behaviour of the function for the viscosity, Eq. 13. Therefore, further experimental measurements of the physical properties would be required to determine the validity of the correlations and there uncertainty outside their regions of interpolation.

In applying the Dittus-Boelter correlation to the mean temperature and mean velocity and the corresponding correlations, a maximum uncertainty in the Nusselt number of $\pm 11 \%$ was obtained. Note that the Dittus-Boelter correlation does not account for the effect that the fluid properties will have on the temperature and velocity field. Nevertheless to fully assess the uncertainty of the numerical models, a sensitivity study of the response of the thermal fluid dynamic models to the uncertainties of the model parameters would have to be performed.

\subsubsection{Field plots}

Fig. 8 presents contour plots obtained from the simulations of heat and fluid flow in the 2-D plane and the power distribution in the core. The temperature fields show strong gradients in the region where the jet of cooler salt enters from the inlet duct (see lower downward arrows in Fig. 8). This jet from the inlet (also observed in the velocity field) persists till the core centre and forms a large vortex of an approximate size of the core radius. A hot region next to the hyperboloid side wall forms in the downward flowing part of the vortex. Both the fluid from the jet entering the core and the hot fluid in the large vortex mix poorly and this jet then exits the core region to the outlet duct. Note that the power distribution in the core is slightly skewed by 

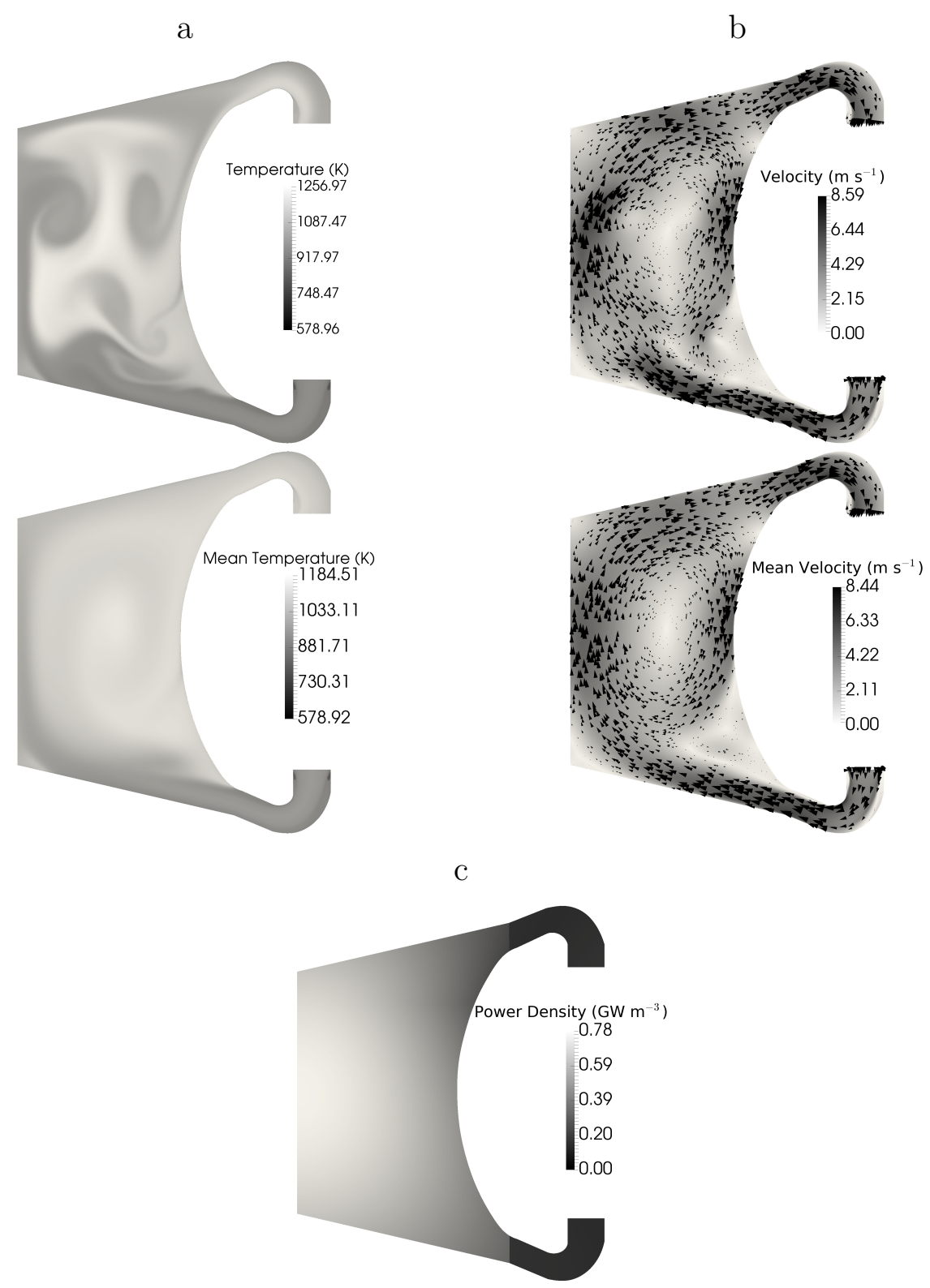

Fig. 8. Contour and vector plots of the instantaneous and mean a) temperature $(\mathrm{K})$ and b) velocity magnitude $\left(\mathrm{m} \mathrm{s}^{-1}\right)$ obtained after $100 \mathrm{~s}$ of simulation time with the frozen wall model from the 2-D slice of the molten salt fast reactor. A contour plot of the power density $\left(\mathrm{GW} \mathrm{m}^{-3}\right)$ applied is plotted in c). 
the spherical function applied to the reactor at heights greater than $1.39 \mathrm{~m}$ above the lower apex of the core barrel base. The power density is negligible in the inlet and outlet pipes, which mimicks with the 3-D coupled simulations performed here. This would not be the case if delayed neutron precursors were modelled as some decay heat would be present in the heat exchanger loop.

The contour plots in Fig. 9 show the cell values of the liquid fraction and magnitude of the heat flux $\left(\mathrm{MW} \mathrm{m}^{-2}\right)$ near to the wall. It can be observed that frozen salt films form in the inlet and outlet ducts (Fig. 9a-b) and two images also show thin frozen salt films at the hyperboloid side wall in the hot zone and near to the inlet entry into the core (Fig. 9c-d). There is also one image of the cells at the base wall (Fig. 9p). All of the images of the liquid fraction at the wall (Fig. 9a-e) show that the frozen salt film is at least $70 \mu \mathrm{m}$ thick (where the distance of the first node at the wall is $10 \mu \mathrm{m}$, which is equivalent to $2 y^{+}$) with the exception of the inlet (Fig. 9a) where the heat flux in the fluid is not exposed to the neutronic heat flux from the core. The corresponding plots of the heat flux show that the flux in the frozen salt in the inlet region is much less than in the core regions. This leads to the formation of thick frozen films that accelerate and direct the flow up the vessels' toroidal wall. The profile of the liquid fraction near to the core wall corresponds to the strong temperature gradient observed there with a high flux at the interface of the solid salt with the molten or mushy salts.

The strong temperature gradient occurs in the molecular diffusion sublayer due to the high Prandtl number of the molten salt simulated here with values of $22.71,19.53$ and 10.22 at $848 \mathrm{~K}, 898 \mathrm{~K}$ and $1,198 \mathrm{~K}$, respectively. This 


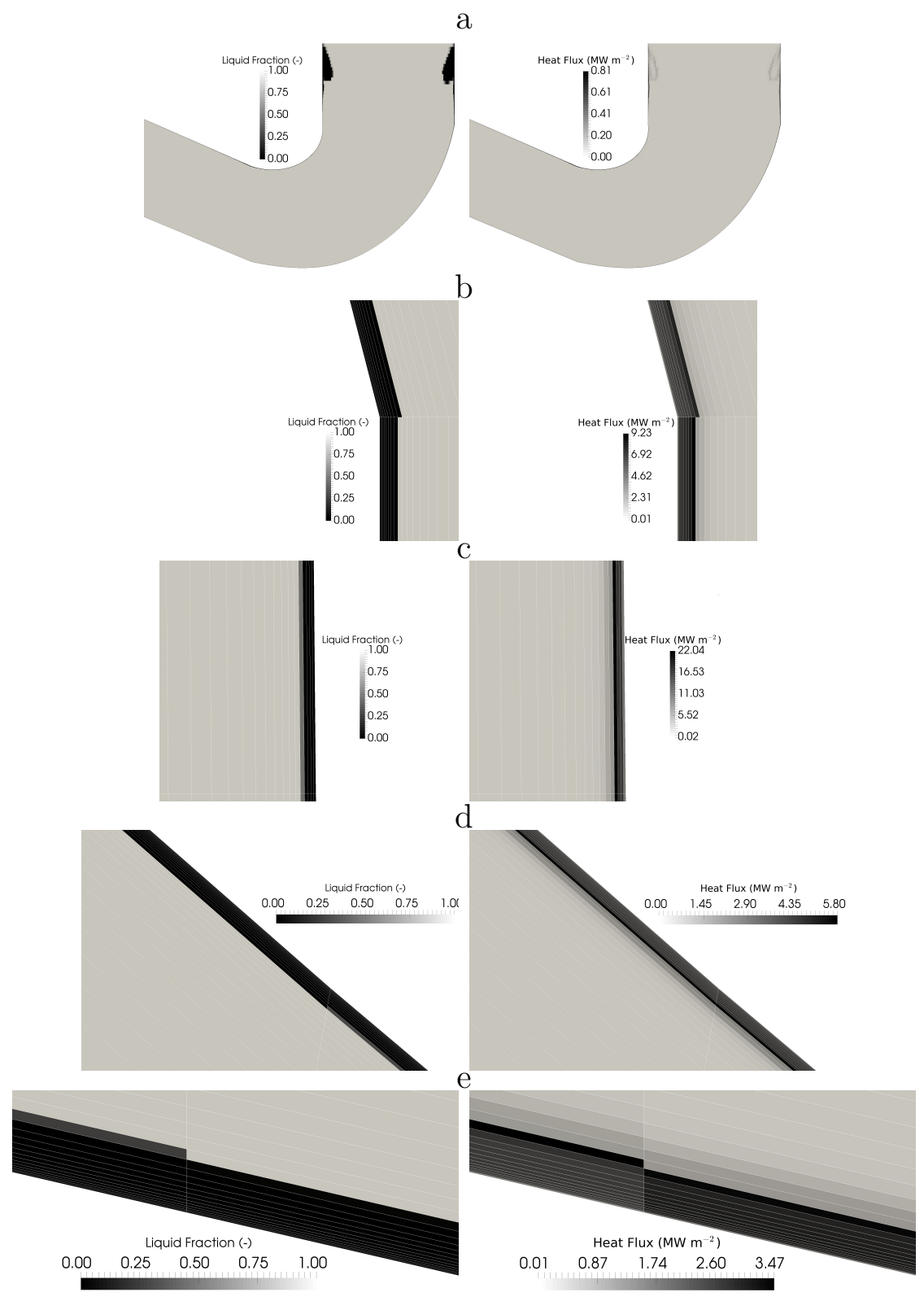

Fig. 9. Contour plots of the liquid fraction (-) and the magnitude of the heat flux given by $\dot{q}=-k \nabla T\left(\mathrm{MW} \mathrm{m}^{-2}\right)$ in different locations obtained after approximately $10 \mathrm{~s}$ of simulation time with the frozen wall model from the 2-D case. a) At the inlet. b) On the inner side of the outlet bend. c) At the midpoint of the core side wall. d) Near to the entrance of the inlet duct to the core barrel. e) On the base wall. 
temperature gradient indicated in Fig. 6 and 9, along with local fluctuations in the temperature due to mixing of the fluid in the core, hinders the formation and stability of the frozen salt film at the wall. The lack of stability of the frozen film is indicated by the fluctuations in the $T^{+}$value (Fig. 5). The variation of $T^{+}$may correspond to fluctuations in the temperature, which is associated changes the physical properties, as $T^{+}=\operatorname{Pry}^{+}$and $y^{+}=y u_{\tau} \rho / \mu$, where $u_{\tau}$ is the friction velocity. The fluctuations in $T^{+}$may also be associated with changes to the temperature gradient leading to changes in the film thickness. $T^{+}$will become larger when hot fast flowing molten salt occurs and this could result in some of the salt being washed away. Therefore, we can conclude that for the selected salt, wall temperature and reactor configuration it will be very difficult to form a stable salt film, which can protect the vessel wall from corrosion when the thickness of the film is less than $1 \mathrm{~mm}$.

\subsection{Conjugate heat transfer applied to the 2-D thermal fluid dynamic calcu-} lations of the MSFR

The heat flux through the reactor walls is modelled heat in simulations of conjugate heat transfer also considering the flow and heat transfer in the reactor and also the heat transfer in the frozen salt. Three additional regions that represented the reactor vessel walls at the bottom, at the top and at the side of the core vessel, on the inlets and outlets and were attached to surfaces that represented isothermal walls of the $102 \mathrm{k}$ mesh cells of the 2-D representation of the MSFR (see Fig. 10. for the location of the vessel walls). These additional regions represented the solid walls and they add $34 \mathrm{k}$ cells to result in a merged mesh of $136 \mathrm{k}$ cells. The vessel wall thickness 
of is $2 \mathrm{~cm}$ and an exponential distribution of 33 nodes was applied from the interface with the fluid of $10 \mu \mathrm{m}$ for the first solid node depth to $3 \mathrm{~mm}$ at the first solid node next to the outer wall. The distribution along the wall matches the distribution on the fluid side. Hastelloy $₫ N$ alloy has been chosen and constant material properties have been assumed $\left(\rho=8,860 \mathrm{~kg} \mathrm{~m}^{-3}, \lambda=\right.$ $21.0 \mathrm{~W} \mathrm{~m}^{-1} \mathrm{~K}^{-1}$ and $c_{p}=578 \mathrm{~J} \mathrm{~kg}^{-1} \mathrm{~K}^{-1}$ ). The external temperature of the vessel was imposed as $573 \mathrm{~K}$, which is an analogue of the case where the wall temperature was set to $623 \mathrm{~K}$ (see $\S 3.3$ ).

Fig. 10a-c shows the velocity, temperature and density fields in the reactor core together with the vessel walls, while Fig. 10 $\mathrm{d}$-f show the distribution of material density, temperature and the magnitude of the heat flux in the wall and in the fluid near to the fluid-solid wall interface. The density plots (Fig. $10 \mathrm{f} \& \mathrm{f}$ ) indicate the locations of the walls, where the density of the nickel alloy is $8,860 \mathrm{~kg} \mathrm{~m}^{-3}$, almost double that of the molten salt.

Note that in the inlet region there is an the absence of the frozen salt blocking the inlet pipe (Fig. 10a-b) in comparison to the simulations depicted in Fig. 5 and Fig. 9. The application of the boundary conditions at the interface between the fluid and solid domains differs to that of the isothermal boundary conditions of the earlier simulations in $\S 3.3$. In the conjugate heat transfer case, the temperature of the vessel wall is affected by the conduction of heat in the solid. The temperature is therefore not constant and a non-uniform heat flux occurs that hinders the formation of the frozen salt film. The absence of the blockage leads to a flow of cold fluid moving up through the centre of the core, which results in the formation of a large recirculation zone next to the core side wall in which hot fluid is observed. Some colder fluid manages 
a

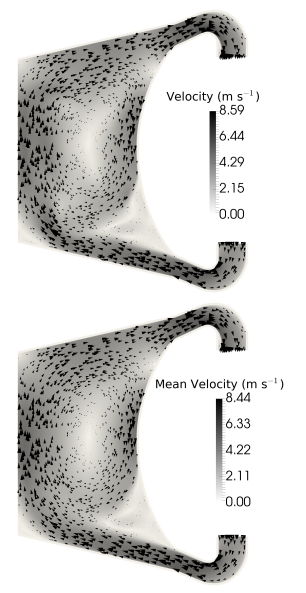

d

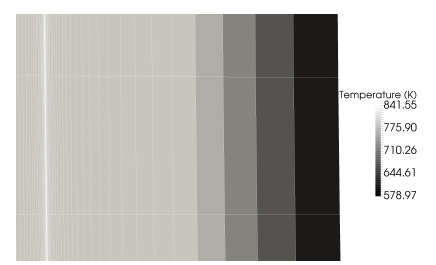

b
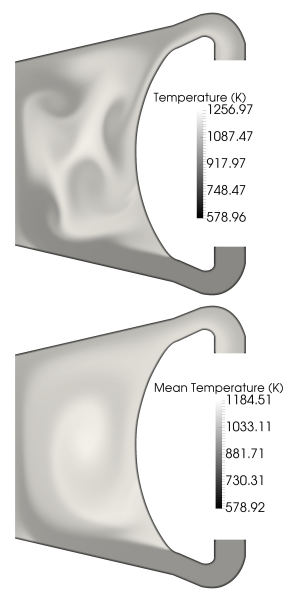

e

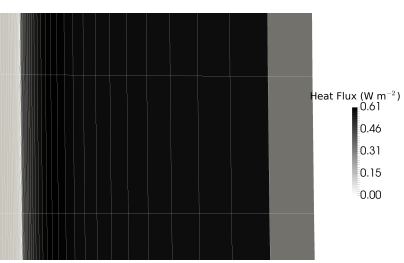

C

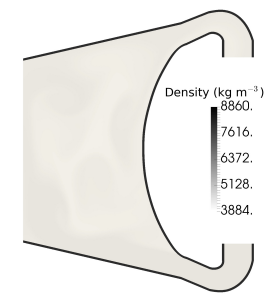

f

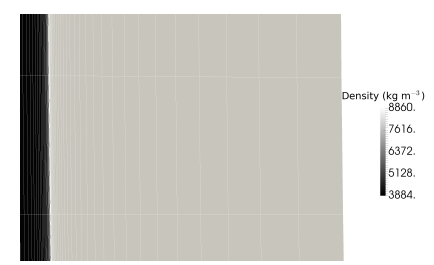

Fig. 10. Contour fields for 2-D flow with conjugate heat transfer with a 20 $\mathrm{mm}$ wall obtained after $75 \mathrm{~s}$ with statistics collected for $25 \mathrm{~s}$. a) Velocity magnitude field $\left(\mathrm{m} \mathrm{s}^{-1}\right)$ in the whole reactor vessel with vectors indicating the flow direction for instantaneous and mean values. b) Temperature field (K) in the reactor vessel for instantaneous and mean values. c) Instantaneous density field $\left(\mathrm{kg} \mathrm{m}^{-3}\right)$ in the reactor vessel. d) Instantaneous temperature field at the vessel wall (K). e) Instantaneous heat flux field $\left(\mathrm{MW} \mathrm{m}^{-2}\right)$ at the vessel wall. f) Instantaneous density field $\left(\mathrm{kg} \mathrm{m}^{-3}\right)$ at the vessel wall. 
to penetrate into the region next to the wall, but this is insufficient to aid the formation of the frozen wall. However, at the core-vessel interface on the narrowest point in the core barrel, it is clear that temperatures were observed that were above the solidification point for the salt (i.e. $841 \mathrm{~K}$ ). The heat penetrated into the vessel wall preventing the formation of the frozen salt film (Fig. 10d). The heat flux is at least two orders of magnitude less than the simulations where the isothermal wall condition was applied confirming that the solidification of the the salt does not occur when the heat flux through the vessel wall is considered.

\subsection{Verification of the coupling of DYN3D-MG to Code_Saturne via MUI- cylindrical test case}

The aim of the simulations performed in this case study is to verify that the neutronics simulation is coupled with the thermal hydraulics and responds to the local changes in temperature as the molten salt with the properties defined by Eq. (11) to Eq. (14) is heated. Here we focus on the effect of the coupling, we have ignored aspects of neutronics modelling that are considered to have an effect on the solution, but complicate the system modelled and may mask the response of neutronics to the thermal fluid dynamics or may have limited impact in such a test system. These aspects include the transport of delayed neutron precursors and heating of the walls from gamma radiation, which is of limited validity of the photon data in the JEFF-3.1.1 nuclear data library used here. We therefore do not include any melting and solidification models or conjugate heat transfer through the walls. We also accept that the hexahedral nodes applied with DYN3D-MG, as described in $\S 3.1$, will provide a numerical model where leakage of the neutrons may be larger than 
the Monte Carlo based solutions by about $200 \mathrm{pcm}$.

The cylindrical core geometry given in $\S 3.1$ had a subcritical $k_{\text {eff }}$ (see Fig. 11). Therefore, a taller geometry of $3.04 \mathrm{~m}$ with $k_{\text {eff }}=1.00007$ at $898 \mathrm{~K}$ was selected. The core modelled in DYN3D-MG used the same number and distribution of nodes as given in $\S 3.1$, except that the height of the nodes in the vertical direction was nearly doubled to $13.2174 \mathrm{~cm}$. Seventeen sets of the cross sections homogenized into 27 energy groups of the ANL distribution by SERPENT are provided across the range of temperatures expected in the MSFR-EVOL (Leppänen, 2017; Cartland-Glover et al., 2018b). DYN3D-MG was operated in the steady mode and coupled to Code_Saturne following the procedures in $\S 2.7$.

The fluid dynamic model had a cylindrical geometry bounded with isothermal (898.15 K), no-slip side walls that had the same dimensions. The top surface was treated as an outlet and the lowest surface was treated as an inlet condition with a uniform inlet velocity and temperature (898.15 K). The mean vertical velocity at the inlet, $\bar{U}_{z, \text { Inlet }}=Q_{T O T} /\left(\rho c_{p} A_{\text {Inlet }} \Delta T\right)$, was determined according to the vertical velocity at the inlet, $U$, the reactor power, $Q_{T O T}$, applied in DYN3D-MG, the expected temperature change, $\Delta T=100 \mathrm{~K}$, the inlet area, $A_{\text {Inlet }}$, with the density and specific heat capacity defined at the inlet temperature from Eq. (11) and Eq. (13), respectively. The $k-\varepsilon$ turbulence model was used with two layer wall functions to allow comparisons with other neutronics and thermal hydraulic solvers.

A coupled simulation was performed with $Q_{T O T}$ set to $3 \mathrm{GW}$ of thermal energy in order to test the response of the coupled codes to the power density and temperature distribution in the cylinder. The outer wall of the neutronics 
models were treated as void conditions.

A comparative calculation using the master version of GeN-Foam (Fiorina et al., 2015) was performed with the same number of energy groups and with the parameters which characterise the delayed neutron precursors removed from the cross sections. Note that a reflective boundary condition was applied to the wall for the neutronics part of the GeN-Foam model and that a fixed uniform hydraulic pressure is applied to the outlet condition and the high Reynolds number formulation of wall functions of OpenFOAM.

The resolution of cylindrical mesh used in both Code_Saturne and GeN-Foam to model the fluid flow was as near as possible to the nodal mesh to allow one to one node data exchange. The mesh was split into o-grid blocks, with 336 cells in the horizontal direction. There were 23 cells in the vertical direction giving 7,728 cells in total. This mesh was also used to model the neutronics in GeN-Foam.

Both Code_Saturneand GeN-Foam simulations ran for 1,000s of simulation time, which required 10,000 time-steps of constant size of $0.1 \mathrm{~s}$.

Both Fig. 11 and Fig. 12 depict traces of the mean and maximum temperatures, the nodal power density and $k_{\text {eff }}$ reported by DYN3D-MG, GeN-Foam and Code_Saturne (temperature only). The data obtained from DYN3DMG is indicated by the designation DYN3D-MG/Code_Saturne, while data obtained from Code_Saturne is by associated with the Code_Saturne/DYN3DMG. The tracks of the location of the minimum and maximum values of the power and the maximum temperatire are plotted in Fig. 13 for both simulations. Fig. 14 presents the contours of the velocity magnitude, temperature of the molten salt modelled by Code_Saturne and the power density pushed 

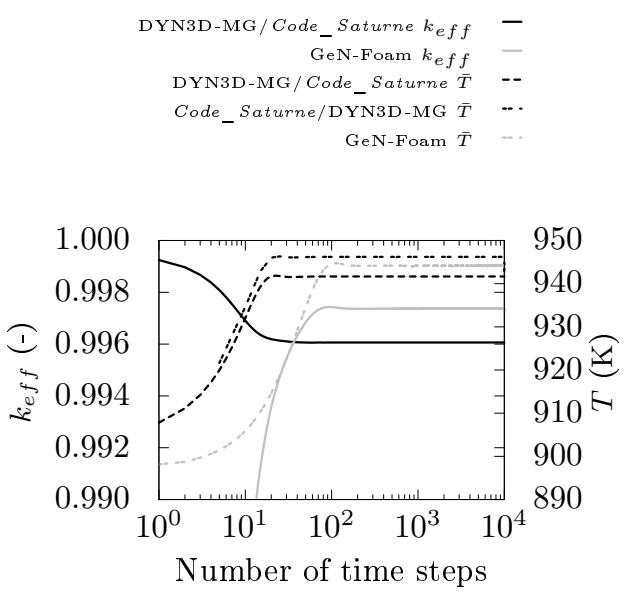

Fig. 11. Traces of the $k_{\text {eff }}(-)$ and mean temperatures $(\mathrm{K})$ obtained by Code_Saturne/DYN3D-MG and GeN-Foam obtained from the coupled simulations of the flow through a cylindrical core with a total power of 3,000 MW. to Code_Saturne via MUI from DYN3D-MG, as well as for GeN-Foam. All the traces in Fig. 11 and Fig. 12 become stable within 100 iterations of the start of the simulation with an increase in the mean temperature. The only observations of changes in the parameters occur while the flow field develops; no other significant changes in these parameters are observed once a steady state is reached. There is a slight reduction in $k_{e f f}$ obtained from DYN3D-MG; however, the $k_{\text {eff }}$ obtained from GeN-Foam started at zero and increased until a similar value was obtained with two cross section sets at 898.15 K and $998.15 \mathrm{~K}$. The average temperatures obtained via coupling the solvers is lower on the DYN3D-MG side than on the Code_Saturne side; this is because a number of nodes at the perimeter of the DYN3D-MG did not meet the nearest neighbour criterion and at these locations the inlet boundary 
a)
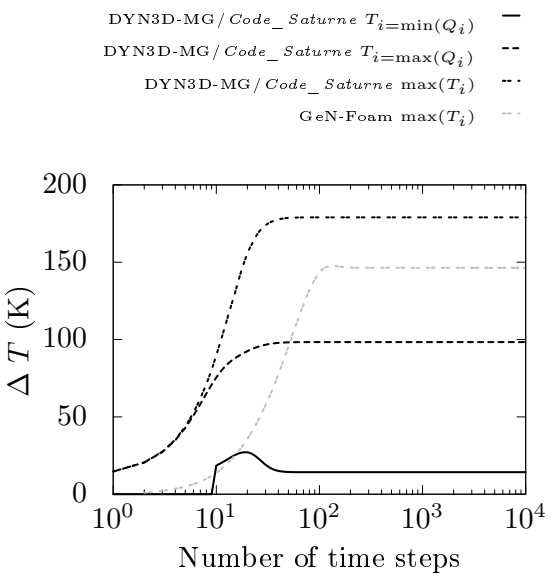

b)
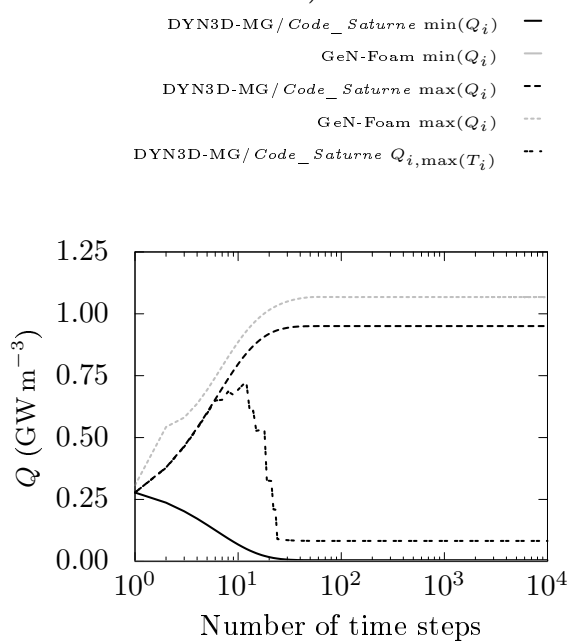

Fig. 12. Traces of the minimum and maximum values of a) maximum temperature change in the core $(\mathrm{K})$ or $\mathrm{b})$ the power density $\left(\mathrm{W} \mathrm{m}^{-3}\right)$ obtained from the coupled cylindrical flow through the core with both Code_Saturne/DYN3DMG and GeN-Foam. The corresponding nodal value of the power density obtained by DYN3D-MG at the maximum temperature and the temperature at the maximum power density are also plotted. The total power was 3,000 MW . 
a) Code_Saturne/DYN3D-MG

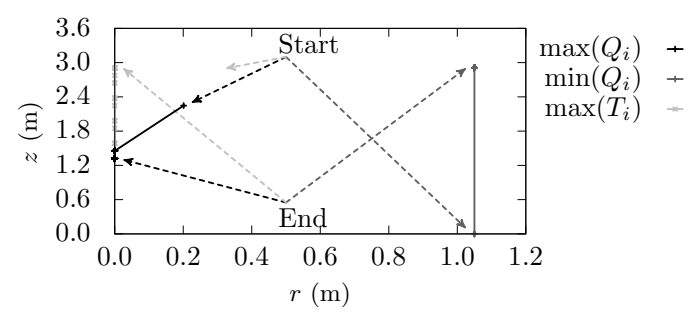

b) GeN-Foam

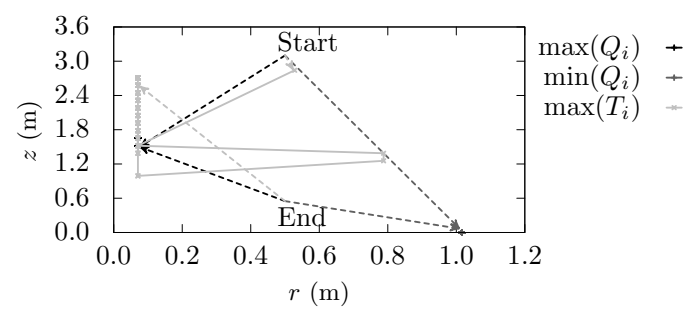

Fig. 13. Tracks of the change in the location of the maximum and minimum of power density $\left(\mathrm{W} \mathrm{m}^{-3}\right)$ and temperature $(\mathrm{K})$ as they vary over the course of the simulation. Simulations were performed with Code_Saturne/DYN3D-MG and GeN-Foam. 
a) Code_Saturne/DYN3D-MG

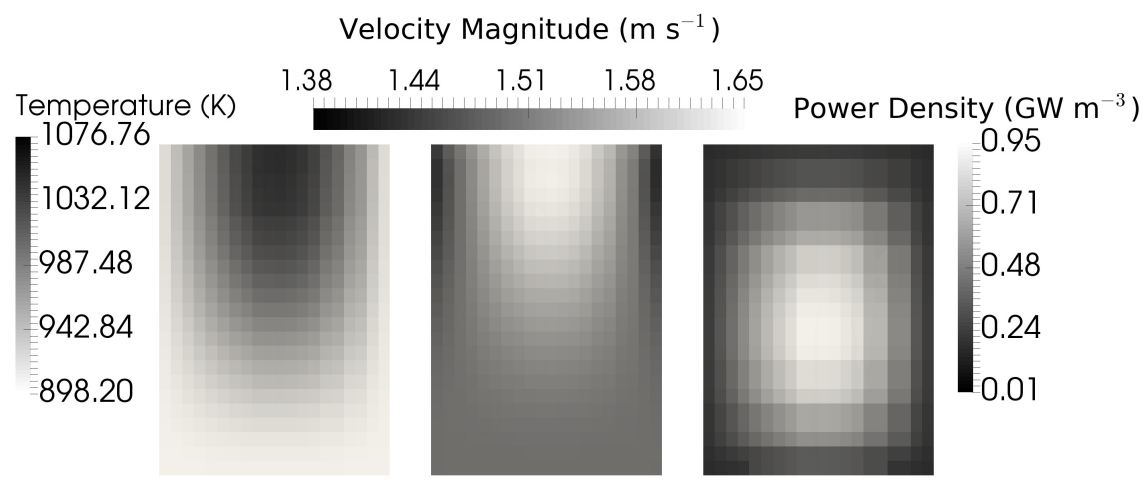

b) GeN-Foam

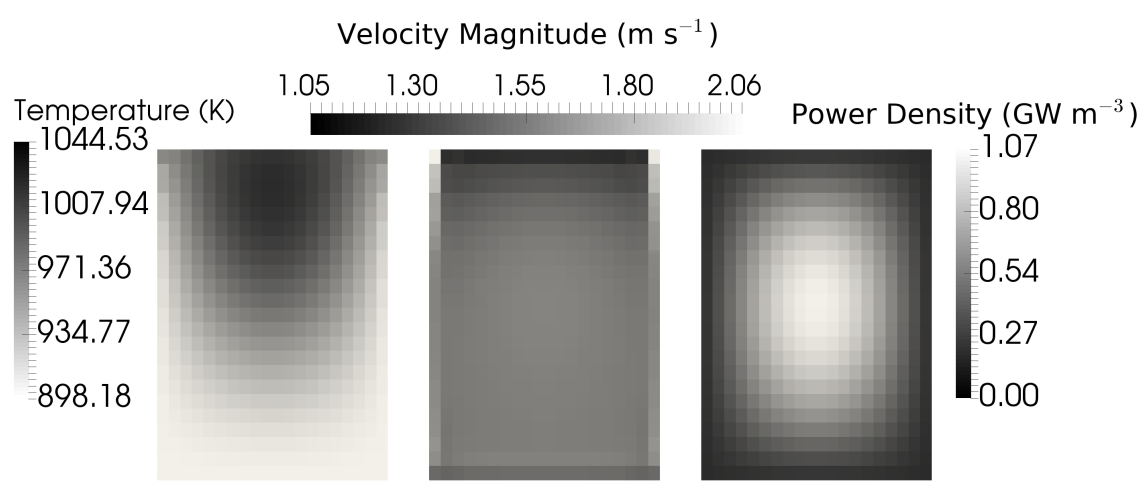

Fig. 14. Contours of temperature $(\mathrm{K})$, velocity magnitude $\left(\mathrm{m} \mathrm{s}^{-1}\right)$ and power density $\left(\mathrm{W} \mathrm{m}^{-3}\right)$ on the mid-plane of the cylrindical MSFR simulations using Code_Saturne/DYN3D-MG and GeN-Foam. 
temperature was applied as a default value. The mean temperature of the GeN-Foam calculation is found in between the curves of obtained from either side of the MUI coupled solvers.

The temperature at the location of the maximum power density is slightly above the desired average temperature increase of $100 \mathrm{~K}$ (see Fig. 12). The maximum temperature observed in the DYN3D-MG and Code_Saturne coupled calculations was approximately $180 \mathrm{~K}$ above the inlet boundary condition temperature and this was at location where a reduced power density was observed. This temperature was over $30 \mathrm{~K}$ higher than the GeN-Foam calculation, which had a maximum temperature that was $146 \mathrm{~K}$ above the inlet temperature, while the maximum power density in the GeN-Foam calculation is significantly larger than the DYN3D-MG calculation. This difference may be due to the nature of the DYN3D-MG, which is hexahedral, while the cylindrical mesh was used in the fluid and neutronics "regions" of the GeN-Foam solver.

The traces of the DYN3D-MG nodal coordinates that were identified by the maximum power and temperature, as well as the minimum power are shown in Fig. 13. This plot helps to explain the change in the temperatures and the corresponding power plotted in Fig. 12, particular the shift in the temperature at the minmum power density at around $10 \mathrm{~s}$, as this location changes from the bottom to the top of the cylinder. The node where the power is greatest on the DYN3D-MG side of the coupling corresponds to a region in the centre of the domain, where the maximum value is observed for the majority of the calculation. The equivalent plot for GeN-Foam shows the maximum power density is located at the midpoint of the cylinder, but the radial position 
is shifted to $5 \mathrm{~cm}$ away from the centreline, which corresponds to the cell centroid of the cylindrical mesh.

Early on in the calculation, i.e. in the first 50 iterations, the maximum power and maximum temperature are located on the same or adjacent DYN3DMG node. The maximum temperature increases further, as the calculation proceeds and the location of this value moves to the top of the cylindrical model. A reduction in the power density is observed in this region, as higher temperature cross-sections are used, which results in a corresponding drop in the neutron reaction rate (Fig. 2).

The lowest power node corresponds to nodes with the lowest temperature, which are located at the wall boundaries, where isothermal boundary conditions were applied in the Code_Saturne simulation. Contour plots of the fluid temperature, velocity and the power density obtained after $1000 \mathrm{~s}$ of simulation time are given in Fig. 14 confirm the distribution of the temperature and power indicated by the traces reported between Fig. 11 and Fig. 13 . Note that the selection of the uniform hydraulic pressure at the outlet of the GeN-Foam simulation affects the velocity field in this region and it also has a slight effect on the temperature field.

It can be concluded from the behaviour that the neutronics model simulated in DYN3D-MG does indeed respond to the temperature field supplied by Code_Saturne. The coupling procedure applied to simulations of the MSFR were on a nearest node basis, which is only first-order accurate. Nevertheless, this case study verifies that the coupling procedure is able to transfer between DYN3D-MG and Code_Saturne and that both solvers respond to the changes in power and fluid temperature. 
A third-order accurate interpolation scheme based on a radial basis function is being developed to improve the coupling procedure. This scheme may improve how MUI identifies the point cloud on which to perform the interpolation of temperature and power obtained from either Code_Saturne or DYN3D-MG. The validation of numerical models accounting for the neutronics and thermal fluid dynamics of a molten salt reactor would require fundamental studies of a zero-power reactor with a homogeneous fuel-salt distribution. This study would provide data for the generation of nuclear data in homogeneous solid and liquid systems. This nuclear data and the numerical models validated on zero power experiments could then be applied for the simulation of powered reactors, where detailed measurements are limited to relatively benign regions.

\subsection{Three-dimensional coupled neutronic-fluid dynamic model of an MSFR with frozen salt films}

A coupled neutronic and thermal fluid dynamic three-dimensional simulation of a hyperboloid MSFR core with 16 inlet and outlet ducts has been performed with the aim of observing the impact of the heating driven by the heat source estimated by the neutronics solver on the formation of the frozen film. The assumptions and uncertainties discussed in $\S 3.3$ and $\S 3.5$ apply to the system modelled here. Note that the conjugate heat transfer through the vessel walls is not considered.

The 2-D profiles of the inlet velocity, temperature and turbulence parameters were obtained from periodic simulations of fully developed flow through a 2-D trapezoidal extrusion of the cross-section of one of the 16 inlet ducts of the MSFR core. The mean inlet velocity $\left(3.82 \mathrm{~m} \mathrm{~s}^{-1}\right.$ and the isothermal wall temperature $(623 \mathrm{~K})$ corresponded to the 2 -D plane simulations reported 
in $\S 3.3$, while the outlets were defined with a zero-gradient condition for temperature and momentum and a Dirichlet condition for the pressure.

The mesh for the thermal fluid dynamic model had 5 million hexahedral elements with a first cell height of $25 \mu \mathrm{m}$. This gave $y^{+}<2$ and a $T^{+}<70$ at the first cell centre. The maximum cell size was $50 \mathrm{~mm}$, which was found where there were o-grid boundaries at the top and bottom of the core barrel. The minimum cell angle was $24^{\circ}$. Each inlet and outlet duct had a minimum cell angle of $21.78^{\circ}$ at the o-grid boundaries that were located near to the wall. The maximum volume change between adjacent cells was less than 11 with large aspect ratio elements were also found near to the wall.

A three-dimensional representation of the core was implemented in the DYN3D-MG model; the model had 637 "homogeneous fuel assemblies" with a width of $10.0570 \mathrm{~cm}$ and 37 vertical layers with a width of $6.9565 \mathrm{~cm}$ giving 23,569 "nodes". The core power was set to $3 \mathrm{GW}$. Cross sections with the ANL distribution of 27 energy groups (Leppänen, 2017) that were obtained from the five regions described in Cartland-Glover et al. (2018b) and they were used to characterise the neutronic behaviour of the MSFR. Two were used to model the fuel salt in the core region, one was a combination of fissile fuel, vessel wall and reflector to model the vessel wall and the remaining cross-sections are used to model the core reflectors, where the media was LiF that contained no fissile material (see the medium grey regions in Fig. 1). This resulted in a $k_{\text {eff }}=1.011318$ in an uncoupled simulation at a temperature of $623.15 \mathrm{~K}$. This neutronic model of the MSFR was coupled to the fluid dynamic model. The $k_{\text {eff }}$ was $0.0059(-0.58 \%)$ below the equivalent value calculated with SERPENT (see the profile plot in Fig. 1). Fig. 15a-c presents 
a

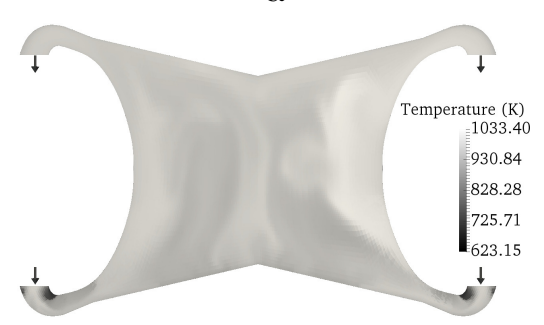

$\mathrm{C}$

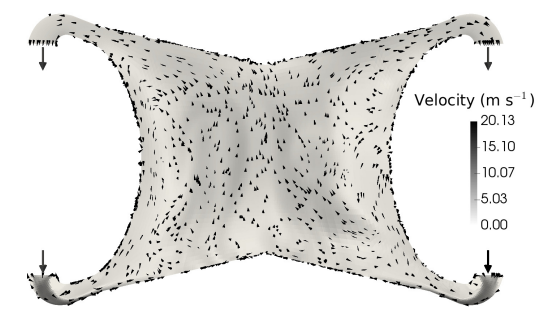

e

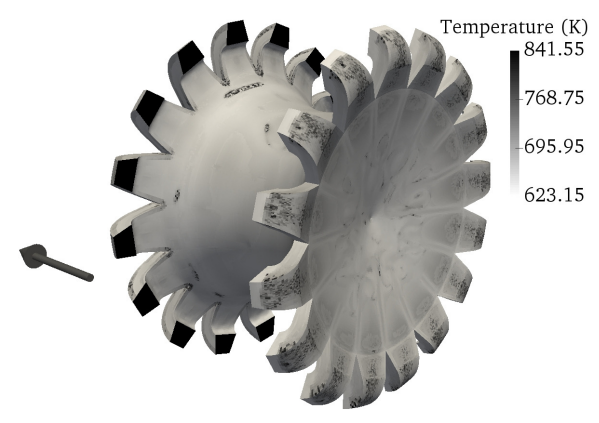

b

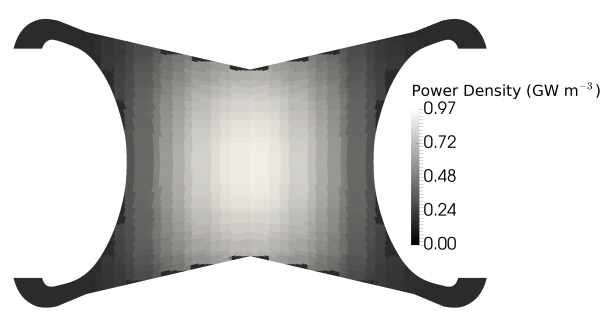

d

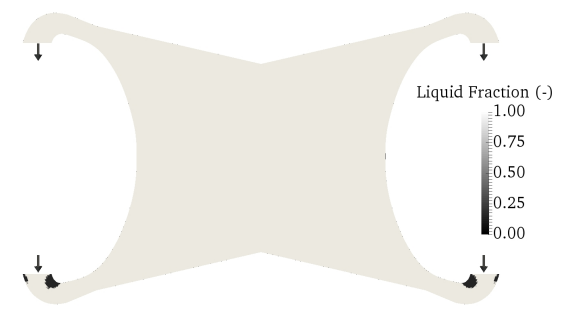

f

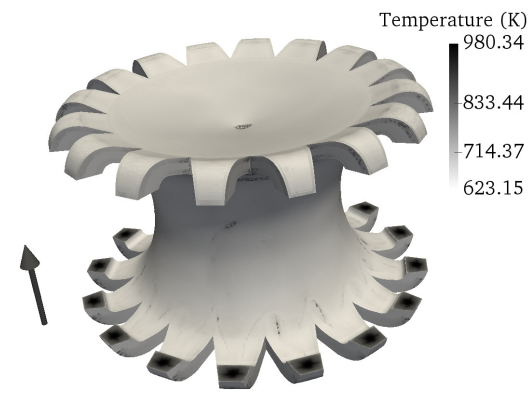

Fig. 15. Mid plane contour plots of a) temperature (K), b) power density $\left.\left(\mathrm{GW} \mathrm{m}{ }^{-3}\right) \mathrm{c}\right)$ velocity $\left(\mathrm{m} \mathrm{s}^{-1}\right)$ with cones indicating the flow direction, and d) liquid fraction (-) at the midplane. The black vectors indicate the direction of the flow into and out of the horizontal planes, which represent the core inlets and outlets; e-f: Outer surface plots of the temperature scaled between the wall boundary condition $623.5 \mathrm{~K}$ and the liquidus point $841.55 \mathrm{~K}$. Here the black vector indicates the positive vertical direction. Note that the solidus point was $814.22 \mathrm{~K}$. f) also indicates inlet temperature profiles. 
contour plots of the temperature, power density, velocity and liquid fraction plotted on the midplane of the MSFR and crossing the centre line of the inlets and outlet ducts. This simulation reached approximately $27 \mathrm{~s}$ of simulation time with the flow nearly fully developed. The frozen salt model was switched on after $13 \mathrm{~s}$ of simulation time. It took four weeks of calculation runs on up to 792 cores of the Cirrus cluster to reach $27 \mathrm{~s}$ of simulation time.

The flow structures observed in the midplane contour plots (Fig. 15a-c ) are similar in form to the 2-D case with the exception of the downward flowing portions of the larger scale vortices, which have a smaller magnitude than the $2-\mathrm{D}$ simulation given in $\$ 3.3$. The distribution of the power compares well with the distribution for the 2-D cases plotted in Fig. 8c, though the maximum power is a little higher in the core centre. Note that the effect of the distribution of the hexahedral nodes used in DYN3D-MG is apparent in the wall regions with negligible levels of power assigned to some of the fluid cells. This can be overcome by the use of better interpolation algorithms (radial basis functions) or the via application of unstructured mesh neutronics solvers. Regardless of the power supplied to the cells at the wall, regions of high temperature form at the walls in these regions, as was the case in the 2-D simulation.

A thick frozen salt film is also formed near to the inlet, which narrows the inlet jet. Otherwise, only a very thin film of frozen salt forms at the wall that is at most $100 \mu \mathrm{m}$ thick in the short time that the frozen wall was simulated. This leads to the flow structures formed in the 3-D simulation differing from the structures presented by Rouch et al. (2014) in that the vortex formed in the entrance to the core barrel for these cases and the 2-D simulations 
performed in 3.2 do not form in the $3-\mathrm{D}$ simulation due to the presence of the frozen salt film forming near to the inlet.

The three-dimensional plots (Fig. 15d-e) of the outer surface temperature indicate regions where the frozen salt film is at its weakest. The regions of warmer colours indicate where the mushy zone is in the first cell next to the wall. These regions are found at the core outlet joins, the side walls, and in regions where hot fluid reaches the wall (see the hot spot on the core barrel). The thick frozen salt films in the inlets can be explained by two factors, the distribution of power in the core (Fig. 15b) and inlets where no heat is supplied and the inlet temperature profiles as indicated in Fig. 15: where temperatures below the solidus temperature were applied near to the wall.

A further simulation was started from the same data point with a radial limit of $1.6 \mathrm{~m}$ for wall temperatures of $623 \mathrm{~K}$. At radii greater than $1.6 \mathrm{~m}$ a temperature of $898 \mathrm{~K}$ was applied. The application of the cooling to the core region greatly reduced the amount of frozen salt formed in the core inlet ducts as shown in Fig. 16. A comparative image from the first simulation is also plotted in Fig. 16. The images show threshold plots of the fraction of liquid between the limits 0 and 0.25 , which indicate both the solid phase and some of the mushy zone. The temperatures experienced by the frozen and mushy zone salt are plotted on the surfaces of the threshold. Note that along-side the temperature plot for the cooled inlets case is a plot of the magnitude of the heat flux on the surface of the salt film is given. The contours plotted on the inner side of the threshold in the mushy zone limit show large and often sharp variations in the temperature. These variations correspond to the local 


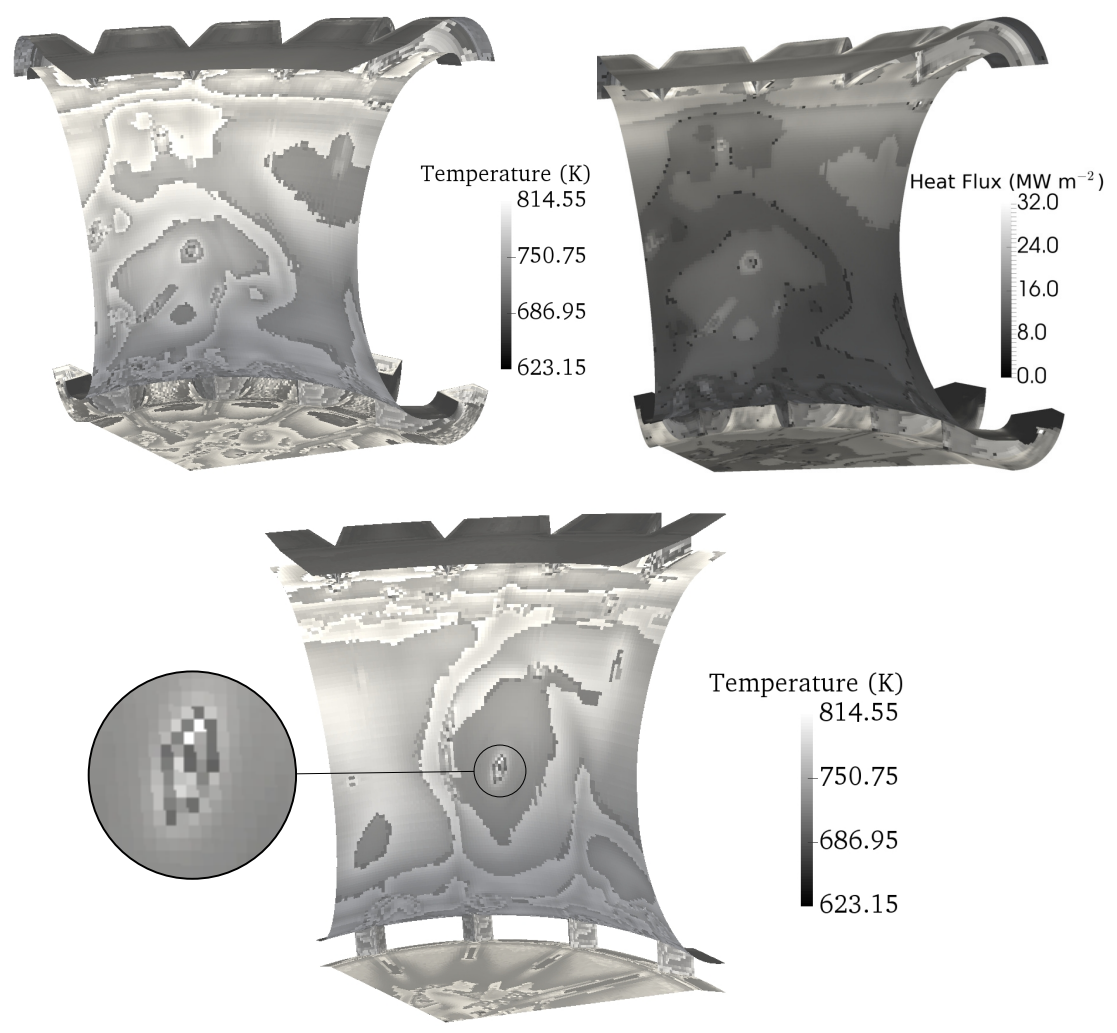

Fig. 16. Cells of the mesh with liquid fraction between 0 and 0.25 obtained from simulations of 3D coupled flow and heat transfer cases. Upper left: Cells coloured by instantaneous temperature whne the inlets are cooled to $623 \mathrm{~K}$ after $28 \mathrm{~s}$ of simulation time; Upper right: Cells coloured by heat flux $\mathrm{MW} \mathrm{m}^{-2}$ for the inlets cooled to $623 \mathrm{~K}$ after $28 \mathrm{~s}$ of simulation time; Lower: Cells coloured by temperature for the core walls cooled $623 \mathrm{~K}$ to a core radius of $1.6 \mathrm{~m}$ and $898 \mathrm{~K}$ in the inlet and outlet ducts. This was obtained after 17.95 s of simulation time; Thick black arrows indicate flow direction. The zoomed circle indicates the region where a hot spot forms and the salt is molten in the location of the white cells. 
momentum and thermal fields of the molten salt, which are not shown here for reasons of clarity. Note that there are several small hots spots in the lower plot of Fig. 16 where the frozen salt film did not cover the wall surface, the largest of which is circled. The hot spots correspond to the flow structures, which form in the core region, where hot fluid accumulates at the wall. In regions where the salt accumulates the heat flux (i.e. in the inlets) the heat flux on the surface of the salt film is much lower.

\section{Recommendations}

Coupled simulations of the thermal fluid dynamics and neutronics of a molten salt fast reactor that study the melting and solidification of salt on cooled vessel walls have been performed. A model for the freezing of the salt on cold vessel structures was implemented and compared against an earlier numerical study of the solidification process. Two-dimensional simulations of the molten salt fast reactor allowed us to determine the mesh resolution required at the wall to model the accumulation of salt in a frozen film and the impact that boundary conditions have on salt film formation. The coupled neutronics and thermal fluid dynamics simulations of a molten salt fast reactor have shown that a very thin layer of frozen salt can form at the wall of the reactor vessel when isothermal wall conditions are applied; however, 2-D uncoupled simulations of the conjugate transfer of heat through the vessel wall show that the frozen film does not form. The reasons why the frozen film do not form can be attributed to the high heat flux arising from the nuclear reactions and the thin thermal boundary sublayer in the core region, which can be attributed to the high Prandtl numbers of the selected fluid. 
Further investigations with refined meshes for the three-dimensional coupled simulations are in the process of being performed in order to fully apply best practice guidelines. In doing so, further developments to the coupling of the thermal fluid dynamics and neutronics are required. This involves the implementation of an interpolation scheme that applies a radial basis function to ensure that the data exchanged is conservative. Future 3-D calculations of the MSFRs should also consider conjugate heat transfer with the vessel wall and coolant media in order to properly assess the non-uniform heat flux on the vessel structures.

The validation of the numerical models applied here for the melting and solidification of molten salts against a database of turbulent convection in cavitites and channel flows in the form of empirical data and direct numerical simulation will be considered in future work. We recommend that for any future study of the solidification of molten salts, a detailed characterisation of the physical properties of the liquid and solid phases of the salt studied should be performed along with the characterisation of the coefficients of momentum loss as well as the trend for temperature and porosity between the liquidus and solidus temperatures. The assessment of the physical properties may also include the variation of different components of the salt mixture and potentially the evolution of the fission product composition during fuel burnup and the effect that this has on the physical properties as the composition moves away from the eutectic point in order to model the long-term operation of the reactors.

Other effects not considered here, which may influence neutronics include the transport of delayed neutron precursor and the gamma heating of the vessel 
walls, as well as the effect that transients in the thermal fluid dynamics have on the neutronics; however, as the greater uncertainty is in the nuclear data upon which the neutronics calculation are based and obtained from inhomogeneous light water reactor systems, it is vital obtain physically relevant nuclear data from appropriate homogeneous system zero-power experiments in order to validate any coupled thermal fluid dynamics and neutronics model of molten salt reactors against these experiments or those of a prototype reactor experiments.

\section{Acknowledgements}

The work presented here was funded by the EPSRC through the Feasibility Study in Energy Research scheme (Ref: EP/R001618/1). David R. Emerson acknowledges additional support from the grants EP/N016602/1 and EP/N033841/1. Gregory Cartland-Glover also acknowledges additional support from the Future Emerging Technologies funding scheme of the European Unions Horizon 2020 research and innovation programme under grant agreement No 671564 .

We acknowledge the support and comments of the developers of Code_Saturne and DYN3D/DYN3D-MG in the studies performed here. Computing resources used in performing the Monte Carlo neutronics calculations were provided by STFC Scientific Computing Department's SCARF cluster http://www.scar f.rl.ac.uk/). The University of Liverpool cluster was used in the development of the nodal neutronic diffusion models (https://www.liverpool.ac.u k/csd/advanced-research-computing/facilities/high-performance-co mputing/). 
We also acknowledge use of Hartree Centre resources in the development of fluid dynamic models and the coupled simulations (http://community.hart ree.stfc.ac.uk/wiki/site/admin/home.html). The coupled simulations were performed on the Cirrus UK National Tier-2 HPC Service at EPCC (http://www.cirrus.ac.uk) funded by the University of Edinburgh and EPSRC (EP/P020267/1). Access to Cirrus was obtained via an EPSRC RAP-Tier 2 allocation.

Ahmad, N., Rappaz, J., Desbiolles, J.L., Jalanti, T., Rappaz, M., Combeau, H., Lesoult, G., Stomp, C., 1998. Numerical simulation of macrosegregation: a comparison between finite volume method and finite element method predictions and a confrontation with experiments. Metallurgical and Materials Transactions A 29, 617630.

Archambeau, F., Méchitoua, N., Sakiz, M., 2004. Code Saturne: A Finite Volume Code for the computation of turbulent incompressible flows - Industrial Applications. International Journal on Finite Volumes 1, http://www.latp.univ-mrs.fr/IJFV/spip.php?article3. URL: https : //hal.archives-ouvertes.fr/hal-01115371.

Aufiero, M., Cammi, A., Fiorina, C., Leppänen, J., Luzzi, L., Ricotti, M.E., 2013. An extended version of the SERPENT-2 code to investigate fuel burn-up and core material evolution of the molten salt fast reactor. Journal of Nuclear Materials 441, $473-486$.

Aufiero, M., Cammi, A., Geoffroy, O., Losa, M., Luzzi, L., Ricotti, M.E., Rouch, H., 2014. Development of an OpenFOAM model for the molten 
salt fast reactor transient analysis. Chemical Engineering Science 111, 390 - 401. doi:10.1016/j.ces.2014.03.003.

Betzler, B.R., Powers, J.J., Worrall, A., 2017. Molten salt reactor neutronics and fuel cycle modeling and simulation with SCALE. Annals of Nuclear Energy 101, 489 - 503. doi:10.1016/j.anucene.2016.11.040.

Billard, F., Laurence, D., 2012. A robust $k-\varepsilon-v^{2} / k$ elliptic blending turbulence model applied to near-wall, separated and buoyant flows. International Journal of Heat and Fluid Flow 33, 45 - 58. doi:10.1016/j.ijheatflui dflow.2011.11.003.

Brovchenko, M., Kloosterman, J.L., Luzzi, L., Merle, E., Heuer, D., Laureau, A., Feynberg, O., Ignatiev, V., Aufiero, M., Cammi, A., Fiorina, C., Alcaro, F., Dulla, S., Ravetto, P., Frima, L., Lathouwers, D., Merk, B., 2019. Neutronic benchmark of the molten salt fast reactor in the frame of the evol and mars collaborative projects. European Journal of Physics - Nuclear Sciences and Technologies 5, 1-26. doi:https://doi.org/10.1051/epjn /2018052.

Capelli, E., 2016. Thermodynamic characterization of salt components for molten salt reactor fuel. Ph.D. thesis. Delft University of Technology. doi:10.4233/uuid:7c2543bf-aec1-4e31-b80e-60f40e34bf66.

Cartland-Glover, G., Rolfo, S., Litskevich, D., Skillen, A., Emerson, D., Merk, B., Moulinec, C., 2019. Resubmitted Figures and Tables from the journal article: "On the numerical modelling of frozen walls in a molten salt fast 
reactor". URL: http://purl.org/net/edata/handle/edata/759, doi:ht tp://dx.doi.org/10.5286/edata/734.

Cartland-Glover, G.M., Rolfo, S., Skillen, A., Emerson, D.R., Moulinec, C., Litskevich, D., Merk, B., 2018a. Modelling frozen salt films in a molten salt fast reactor, in: 26th International Conference on Nuclear Engineering, p. V008T09A038. doi:10.1115/ICONE26-82210. Paper No. ICONE26-82210.

Cartland-Glover, G.M., Rolfo, S., Skillen, A., Emerson, D.R., Moulinec, C., Litskevich, D., Merk, B., 2018b. Modelling the neutronics of a molten salt fast reactor using DYN3D-MG for the investigation of the application of frozen wall technology, in: 26th International Conference on Nuclear Engineering, p. V005T05A024. doi:10.1115/ICONE26-82170. Paper No. ICONE26-82170.

Catalyst Database, 2017. Detail information of $\mathrm{Li}_{3} \mathrm{ThF}_{7}$. URL: http://ww w. catalysthub.net/materials.php?id=1824880. (accessed 15 December 2017).

Cazalbou, J.B., Chassaing, P., Dufour, G., Carbonneau, X., 2005. Twoequation modeling of turbulent rotating flows. Physics of Fluids 17, 055110. doi:10.1063/1.1920630.

Cervi, E., Lorenzi, S., Cammi, A., Luzzi, L., 2019. Development of a multiphysics model for the study of fuel compressibility effects in the molten salt fast reactor. Chemical Engineering Science 193, 379-393.

Duderstadt, J.J., Hamilton, L.J., 1976. Nuclear reactor analysis. John Wiley \& Sons, Inc., New York, USA. 
Fiorina, C., Aufiero, M., Cammi, A., Franceschini, F., Křepel, J., Luzzi, L., Mikityuk, K., Ricottia, M.E., 2013. Investigation of the msfr core physics and fuel cycle characteristics. Progress in Nuclear Energy 68, 153-168.

Fiorina, C., Aufiero, M., Cammi, A., Guerrieri, C., Křepel, J., Luzzi, L., Mikityuk, K., Ricotti, M.E., 2012. Analysis of the MSFR core neutronics adopting different neutron transport models, in: 20th International Conference on Nuclear Engineering and the ASME 2012 Power Conference, pp. 219-228. doi:10.1115/ICONE20-POWER2012-54519. Paper No. ICONE20-POWER2012-54519.

Fiorina, C., Clifford, I., Aufiero, M., Mikityuk, K., 2015. GeN-Foam: a novel OpenFOAM $₫$ based multi-physics solver for $2 \mathrm{D} / 3 \mathrm{D}$ transient analysis of nuclear reactors. Nuclear Engineering and Design 294, 2437.

Flageul, C., 2016. Verification report of Code_Saturne 4.0 Internal Coupling. Technical Report. EDF SA.

Fournier, Y., Bonelle, J., Moulinec, C., Shang, Z., Sunderland, A., Uribe, J., 2011. Optimizing Code_Saturne computations on petascale systems. Computers \& Fluids 45, 103 - 108. doi:10.1016/j.compfluid.2011.0 1.028. 22nd International Conference on Parallel Computational Fluid Dynamics (ParCFD 2010).

Gilbert, R.A., 1962. Heat of fusion of $3 \mathrm{LiF} \cdot \mathrm{ThF}_{4}$. Journal of Chemical \& Engineering Data 7, 388-389. doi:10.1021/je60014a021.

Gill, M., 2016. The potential impact of fast reactors and fuel recycling schemes on the UKs nuclear waste inventory. Ph.D. thesis. University 
of Manchester. URL: https://www.escholar.manchester.ac.uk/uk-acman-scw: 296742 .

Grahn, A., Gommlich, A., Kliem, S., Bilodid, Y., Kozmenkov, Y., 2017. Simulation of an MSLB scenario using the 3D neutron kinetic core model DYN3D coupled with the CFD software Trio_U. Nuclear Engineering and Design 315, 117 - 127. doi $10.1016 / j$.nucengdes.2017.02.002.

Grahn, A., Kliem, S., Rohde, U., 2015. Coupling of the 3D neutron kinetic core model DYN3D with the CFD software ANSYS-CFX. Annals of Nuclear Energy 84, 197 - 203. doi:10.1016/j .anucene.2014.12.015.

Hannaford, B.A.., McNeese, L.E., 1969. Continuous fluorination of molten salt, in: Rosenthal, M.W., Briggs, R., Kasten, P.R. (Eds.), Molten-Salt Reactor Program Semi-annual progress report. Oak Ridge National Laboratory. ORNL-4344. chapter 26, pp. 302-305.

Heller, H.P., Schürmann, M., Scholl, K., Haustein, N., Lychatz, B., Falkus, J., 2017. Calibration problems with the viscosity measurement of liquid metallurgical slags. Journal of Physics: Conference Series 790, 012010. doi:10.1088/1742-6596/790/1/012010.

Hérbert, A., 2009. Applied reactor physics. Presses internationales Polytechnique, Quebec, Canada.

Heuer, D., Merle-Lucotte, E., Allibert, M., Brovchenko, M., Ghetta, V., Rubiolo, P., 2014. Towards the thorium fuel cycle with molten salt fast reactors. Annals of Nuclear Energy 64, $421-429$. 
Ignatiev, V., Feynberg, O., Merzlyakov, A., Surenkov, A., Zagnitko, A., Afonichkin, V., Bovet, A., Khokhlov, V., Subbotin, V., Fazilov, R., Gordeev, M., Panov, A., Toropov, A., 2012. Progress in development of MOSART concept with th support, in: 2012 International Congress on Advances in Nuclear Power Plants, pp. 943-952.

Ignatiev, V., Surenkov, A., 2013. Alloys compatibility in molten salt fluorides: Kurchatov institute related experience. Journal of Nuclear Materials 441, 592 - 603. doi:10.1016/j.jnucmat.2013.05.007.

Janz, G.J., Allen, C.B., Bansal, N.P., Murphy, R.M., Tomkins, R.P.T., 1979. Physical Properties Data Compilations Relevant to Energy Storage II Molten Salts: Data on Single and Multicomponent Salt Systems. Technical Report NSRDS-NBS 61, Part II. US Department of Commerce, National Bureau of Standards.

Jareteg, K., Vinai, P., Sasic, S., Demazire, C., 2015. Coupled fine-mesh neutronics and thermal-hydraulics modeling and implementation for PWR fuel assemblies. Annals of Nuclear Energy 84, 244 - 257. doi:10.1016/j anucene.2015.01.037.

Kader, B., 1981. Temperature and concentration profiles in fully turbulent boundary layers. International Journal of Heat and Mass Transfer 24, 1541 - 1544. doi:10.1016/0017-9310(81)90220-9.

Kader, B., 1991. Heat and mass transfer in pressure-gradient boundary layers. International Journal of Heat and Mass Transfer 34, 2837 - 2857. doi: $10.1016 / 0017-9310(91) 90245-A$. 
Kliem, S., Bilodid, Y., Fridman, E., Baier, S., Grahn, A., Gommlich, A., Nikitin, E., Rohde, U., 2016. The reactor dynamics code DYN3D. Kerntechnik 81, 170 - 172. doi:10.3139/124.110692.

Křepel, J., Rohde, U., Grundmann, U., Weiss, F.P., 2007. DYN3D-MSR spatial dynamics code for molten salt reactors. Annals of Nuclear Energy $34,449-462$.

Laureau, A., Aufiero, M., Rubiolo, P.R., Merle-Lucotte, E., Heuer, D., 2015. Coupled neutronics and thermal-hydraulics transient calculations based on a fission matrix approach: application to the molten salt fast reactor, in: Joint International Conference on Mathematics and Computation (M\&C), Supercomputing in Nuclear Applications (SNA) and the Monte Carlo (MC) Method, pp. 1-12.

Laureau, A., Rubiolo, P.R., Heuer, D., Merle-Lucotte, E., Brovchenko, M., 2013. Coupled neutronics and thermal-hydraulics numerical simulations of a molten fast salt reactor (MFSR), in: Array (Ed.), SNA + MC 2013 Joint International Conference on Supercomputing in Nuclear Applications + Monte Carlo, p. 02307. doi:10.1051/snamc/201402307.

Leppänen, J., 2017. Serpent wiki - ANL 27 group structure. URL: http:// serpent.vtt.fi/mediawiki/index.php/ANL_27_group_structure. (accessed 18th December 2017).

Leppänen, J., Pusa, M., Viitanen, T., Valtavirta, V., Kaltiaisenaho, T., 2015. The Serpent Monte Carlo code: Status, development and applications in 
2013. Annals of Nuclear Energy 82, 142 - 150. doi:10.1016/j.anucene. 2014.08 .024

van der Linden, E., 2012. Coupled neutronics and computational fluid dynamics for the molten salt fast reactor. Master's thesis. Delft University of Technology.

MacPherson, H.G., 1985. The molten salt reactor adventure. Nuclear Science and Engineering 90, 374-380. doi:10.13182/NSE90-374.

Mahadevan, V.S., Merzari, E., Tautges, T., Jain, R., Obabko, A., Smith, M., Fischer, P., 2014. High-resolution coupled physics solvers for analysing fine-scale nuclear reactor design problems. Philosophical Transactions of the Royal Society A: Mathematical, Physical and Engineering Sciences 372, 20130381. doi:10.1098/rsta.2013.0381.

Merk, B., Konheiser, J., 2014. Neutron shielding studies on an advanced molten salt fast reactor design. Annals of Nuclear Energy 64, $441-448$. doi:10.1016/j . anucene.2013.07.014.

Merk, B., Litskevich, D., 2015. On the burning of plutonium originating from light water reactor use in a fast molten salt reactora neutron physical study. Energies 8, 12557-12572. doi:10.3390/en81112328.

Merk, B., Litskevich, D., Bankhead, M., Taylor, R.J., 2017. An innovative way of thinking nuclear waste management neutron physics of a reactor directly operating on SNF. PLOS ONE 12, 1-19. doi:10.1371/journal. pone.0180703. 
Pérez Mañes, J., Espinoza, V.H.S., Chiva, S., Stieglitz, R., 2014. A new coupled CFD/neutron kinetics system for high fidelity simulations of LWR core phenomena: Proof of concept. Science and Technology of Nuclear Installations 2014. doi:10.1155/2014/294648.

Pettersen, E.E., 2016. Coupled multi-physics simulations of the Molten Salt Fast Reactor using coarse-mesh thermal-hydraulics and spatial neutronics. Master's thesis. Université Paris-Saclay.

Rasendra, M.A., 2012. Coupled neutronics and computational fluid dynamics for the molten salt fast reactor. Master's thesis. Delft University of Technology.

Rohde, U., Kliem, S., Grundmann, U., Baier, S., Bilodid, Y., Duerigen, S., Fridman, E., Gommlich, A., Grahn, A., Holt, L., Kozmenkov, Y., Mittag, S., 2016. The reactor dynamics code DYN3D - models, validation and applications. Progress in Nuclear Energy 89, 170 - 190. doi:10.1016/j.pn ucene.2016.02.013.

Rouch, H., Geoffroy, O., Rubiolo, P., Laureau, A., Brovchenko, M., Heuer, D., Merle-Lucotte, E., 2014. Preliminary thermalhydraulic core design of the molten salt fast reactor (MSFR). Annals of Nuclear Energy 64, 449 456. doi:/10.1016/j. anucene.2013.09.012.

Santamarina, A., Bernard, D., Rugama, Y., 2014. The JEFF-3.1.1 Nuclear Data Library. Technical Report JEFF Report 22 NEA No. 6807. Nuclear Energy Agency, Organisation for Economic Co-operation and Development. 
Sohal, M.S., Ebner, M.A., Sabharwall, P., Sharpe, P., 2010. Engineering database of liquid salt thermophysical and thermochemical properties. Technical Report INL/EXT-10-18297. Idaho National Laboratory.

Tang, Y.H., Kudo, S., Bian, X., Li, Z., Karniadakis, G.E., 2015. Multiscale universal interface: A concurrent framework for coupling heterogeneous solvers. Journal of Computational Physics 297, 13 - 31. doi:10.1016/j.jc p.2015.05.004.

Tano, M., Rubiolo, P., Doche, O., 2017. Progress in modeling solidification in molten salt coolants. International Journal of Modelling and Simulation in Materials Science and Engineering 25, 074001.

Tunstall, R., Laurence, D., Prosser, R., Skillen, A., 2014. RANS studies of a T-junction: A comparison of turbulence models on low $y^{+}$meshes, in: ETMM10: 10th International ERCOFTAC Symposium on Engineering Turbulence Modelling and Measurements, pp. 1-8.

Voller, V., Prakash, C., 1987. A fixed grid numerical modelling methodology for convection-diffusion mushy region phase-change problems. International Journal of Heat and Mass Transfer 30, 1709 - 1719. doi:10.1016/00179310(87)90317-6.

Yan, J., Kochunas, B., Hursin, M., Downar, T., Karoutas, Z., Baglietto, E., 2011. Coupled computational fluid dynamics and MOC neutronic simulations of westinghouse PWR fuel assemblies with grid spacers, in: 14th International Topical Meeting on Nuclear Reactor Thermal Hydraulics, NURETH-14, pp. 1-15. 
Zhang, D., Liu, L., Liu, M., Xu, R., Gong, C., Qiu, S., 2017. Neutronics/thermal-hydraulics coupling analysis for the liquid-fuel mosart concept. Energy Procedia 127, 343-351.

Zhang, D.L., Qiu, S.Z., Liu, C.L., Su, G.H., 2008. Steady state investigation on neutronics of a molten salt reactor considering the flow effect of fuel salt. Chinese Physics C 32, 624-628.

Zhang, K., Tang, X., Cao, L., 2019. Development and verification of a model for generation of MSFR few-group homogenized cross-sections based on a Monte Carlo code OpenMC. Annals of Nuclear Energy 124, 187-197. 